ARTICLE

\title{
Discovery of first-in-class inhibitors of ASH1L histone methyltransferase with anti-leukemic activity
}

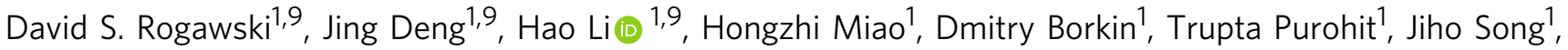
Jennifer Chase ${ }^{2}$, Shuangjiang $\mathrm{Li}^{1}$, Juliano Ndoj ${ }^{1}$, Szymon Klossowski ${ }^{1}$, EunGi Kim${ }^{1}$, Fengbiao Mao ${ }^{1}$, Bo Zhou ${ }^{1}$ James Ropa,3, Marta Z. Krotoska', Zhuang Jin (1) ${ }^{1}$, Patricia Ernst ${ }^{4}$, Xiaomin Feng ${ }^{5}$, Gang Huang (i) ${ }^{5}$, Kenichi Nishioka ${ }^{6}$, Samantha Kelly ${ }^{7}$, Miao He $^{8}$, Bo Wen $^{8}$, Duxin Sun ${ }^{8}$, Andrew Muntean', Yali Dou (D) ${ }^{1}$, Ivan Maillard ${ }^{2,7}$, Tomasz Cierpicki (i) ${ }^{1 凶} \&$ Jolanta Grembecka (i) ${ }^{1 凶}$

ASH1L histone methyltransferase plays a crucial role in the pathogenesis of different diseases, including acute leukemia. While ASH1L represents an attractive drug target, developing ASH1L inhibitors is challenging, as the catalytic SET domain adapts an inactive conformation with autoinhibitory loop blocking the access to the active site. Here, by applying fragment-based screening followed by medicinal chemistry and a structure-based design, we developed first-in-class small molecule inhibitors of the ASH1L SET domain. The crystal structures of ASH1L-inhibitor complexes reveal compound binding to the autoinhibitory loop region in the SET domain. When tested in MLL leukemia models, our lead compound, AS-99, blocks cell proliferation, induces apoptosis and differentiation, downregulates MLL fusion target genes, and reduces the leukemia burden in vivo. This work validates the ASH1L SET domain as a druggable target and provides a chemical probe to further study the biological functions of ASH1L as well as to develop therapeutic agents.

\footnotetext{
${ }^{1}$ Department of Pathology, University of Michigan, Ann Arbor, MI, USA. ${ }^{2}$ Life Sciences Institute, University of Michigan, Ann Arbor, MI, USA. ${ }^{3}$ Department of Microbiology and Immunology, Indiana University School of Medicine, Indianapolis, IN, USA. ${ }^{4}$ Department of Pediatrics, University of Colorado Denver, Anschutz Medical Campus, Aurora, CO, USA. ${ }^{5}$ Department of Pathology and Laboratory Medicine, Cincinnati Children's Hospital, Cincinnati, OH, USA. ${ }^{6}$ Department of Internal Medicine Musashimurayama Hospital, Enoki 1-1-5, Musashimurayama, Tokyo, Japan. ${ }^{7}$ Division of Hematology-Oncology, Perelman School of Medicine, University of Pennsylvania, Philadelphia, PA, USA. ${ }^{8}$ College of Pharmacy, University of Michigan, Ann Arbor, MI, USA. ${ }^{9}$ These authors

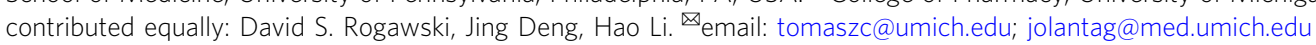


C hromatin modulation through covalent modifications of histones, including histone lysine methylation, plays an important role in the regulation of gene expression both in normal and pathological states ${ }^{1}$. Histone methyltransferases (HMTs) involved in methylation of histone 3 on lysine 36 (H3K36) play an important role as regulators of cell growth, differentiation, stemness, and DNA repair pathways ${ }^{2,3}$. The ASH1L (absent, small, or homeotic-like 1) protein is a histone lysine methyltransferase that catalyzes the mono- and dimethylation of $\mathrm{H} 3 \mathrm{~K} 36$, representing an activating mark on chromatin $^{2,4-6}$. At the molecular level, ASH1L is a large, 3000 amino acid protein that contains a catalytic SET domain responsible for the transfer of a methyl group from the S-adenosyl methionine (SAM) cofactor to the lysine substrate ${ }^{2,7}$. In addition to the SET domain, ASH1L contains three chromatin reader domains, such as bromodomain, $\mathrm{PHD}$ and $\mathrm{BAH}$, as well as a long unstructured region at the $\mathrm{N}$-terminus of the protein ${ }^{2}$.

In mammals, ASH1L shares a conserved functional relationship with another trithorax-group protein Mixed Lineage Leukemia $1(\mathrm{MLL} 1)^{8}$. Both ASH1L and MLL1 positively regulate the expression of HOX genes and support the self-renewal potential of hematopoietic stem cells ${ }^{4,9-12}$. In addition, ASH1L plays a crucial role in the pathogenesis of acute leukemia with chromosomal translocations of the $M L L 1$ gene (also known as KMT2A) ${ }^{9}$, which occur in $5-10 \%$ of acute leukemia patients and lead to very poor prognosis (only $\sim 35 \% 5$-year survival) ${ }^{13-15}$. The knockdown of ASH1L in MLL leukemia cells leads to growth arrest, apoptosis, differentiation, and downregulation of HOXA9 genes that are essential to leukemogenesis, and abrogates development of MLL leukemia in vivo ${ }^{9}$. At the molecular level, ASH1L maintains the H3K36me 2 mark, which enables the recruitment of the MLL1 protein complex to chromatin to activate target gene expression in leukemia cells ${ }^{9}$. In addition, the overexpression of ASH1L was found in thyroid, breast, and liver cancers, and is associated with enhanced cancer cell growth and aggressive disease ${ }^{16-18}$. Thus, the importance of ASH1L in acute leukemia and other cancers strongly supports its high relevance as an attractive therapeutic target in oncology. However, to date no small molecule inhibitors of ASH1L have been reported.

Previous studies have shown that the catalytic SET domain of ASH1L is involved in the regulation of Hoxa genes in mouse embryonic stem (ES) cells ${ }^{5}$, suggesting that the histone methyltransferase activity of ASH1L is important for its gene activating function. Here, we demonstrate the relevance of the catalytic SET domain of ASH1L in the leukemic transformation mediated by MLL fusion proteins, supporting the rationale for inhibitor development. Then, we present the development of the first-inclass small molecule inhibitors of the catalytic SET domain of ASH1L with nanomolar activity and high selectivity to ASH1L. We also report crystal structures of the ASH1L-inhibitor complexes, revealing compounds binding to the autoinhibitory loop region in the SET domain. When tested in MLL leukemia cells, our lead compound, AS-99, blocked proliferation, induced apoptosis and differentiation, downregulated MLL fusion target genes, and reduced the leukemia burden in a mouse model of MLL leukemia. Overall, this work reveals that the ASH1L SET domain represents a druggable target and provides a valuable chemical probe to study the biological functions of ASH1L and a molecular scaffold to develop therapeutic agents targeting ASH1L.

\section{Results}

ASH1L SET domain is required for transformation by MLL fusions. Previous studies have revealed an important role of ASH1L in leukemogenesis mediated by MLL fusion proteins ${ }^{9}$, however, the requirement for the catalytic SET domain in this process was unknown. To address this question, we took advantage of a mouse model with an in-frame deletion of the Ash1l SET domain $(\triangle \mathrm{SET} \text { Ash } 11)^{5}$. Importantly, these mice remain viable, and we isolated bone marrow cells from these mice and from the wild type (WT) control mice and transformed them with two $M L L$ fusion oncogenes, $M L L-A F 9$ and $M L L-A F 6$, as well as with control oncogenes, HOXA9/MEIS1 (HM-2) and E2A$H L F$, the later acts through non-HOX pathways ${ }^{19}$. By performing a colony forming assay with serial replating, we found that the transduction of WT Ash1l bone marrow cells with any of these oncogenes resulted in colony formation after several rounds of replating, which is indicative of transformation (Fig. 1a and Supplementary Fig. 1a). In contrast, both $M L L-A F 9$ and $M L L-$ AF6 oncogenes failed to efficiently transform bone marrow cells isolated from the $\triangle \mathrm{SET}$ Ash1l mice as manifested by a substantially reduced $(>75 \%)$ colony number compared to the WT Ash1l background (Fig. 1a and Supplementary Fig. 1a). Furthermore, the remaining colonies from $\triangle$ SET Ash1l cells transduced with the $M L L$ fusion oncogenes were significantly smaller and more diffused when compared to colonies from the WT Ash1l background (Fig. 1b and Supplementary Fig. 1b). The cells derived from these colonies showed an increased differentiating phenotype and a higher level of myeloid differentiation markers (Ly-6G and Gr-1), supporting the inefficient transformation of $\triangle$ SET Ash1l bone marrow cells by MLL fusion proteins (Fig. 1c and Supplementary Fig. 1d). In contrast, the loss of the Ash1l SET domain did not impact transformation mediated by the HOXA9/ MEIS1 or E2A-HLF oncogenes, as the number and size of the colonies and cell morphology did not differ between WT or $\triangle$ SET Ash1l bone marrow cells (Fig. 1a,b,c and Supplementary Fig. 1a, c). Finally, we performed gene expression studies in $M L L-A F 9$ and $M L L-A F 6$ transformed bone marrow cells and found that genes highly relevant to MLL leukemia (Hoxa5, Hoxa9, Hoxa10, Meis1, and Mef2C) were expressed at much lower levels in the $\triangle$ SET Ash1l versus WT Ash1l cells, (Fig. 1d). In contrast, the expression level of these genes was not markedly different in the bone marrow cells transformed with control oncogenes HOXA9/ MEIS1 or E2A-HLF in the $\triangle$ SET Ash1l versus WT Ash1l cells, Supplementary Fig. 1e. Overall, these results validate the SET domain of ASH1L as essential for MLL-fusion protein mediated leukemogenesis, supporting the rationale for developing small molecules blocking the histone methyltransferase activity of ASH1L.

Identification of a fragment hit that binds to the ASH1L SET domain. To identify ASH1L inhibitors we conducted an NMRbased screening of $\sim 1600$ fragment-like compounds from an inhouse library and identified a thioamide containing compound 1 that binds to the ASH1L SET domain, as validated by chemical shift perturbations on the ${ }^{15} \mathrm{~N}-{ }^{1} \mathrm{H}$ TROSY-HSQC spectrum (Fig. 2a and Supplementary Fig. 2a). In NMR titration experiments, we observed linear chemical shift perturbations up to 1 $\mathrm{mM}$ of 1 , indicating weak binding affinity with $\mathrm{K}_{\mathrm{d}}$ above $1 \mathrm{mM}$ (Fig. 2a). To identify the binding site of $\mathbf{1}$, we mapped chemical shift perturbations onto the crystal structure of the ASH1L SET domain (Fig. 2b). As we previously reported, due to conformational heterogeneity we could not observe signals on the NMR spectra of ASH1L corresponding to the autoinhibitory loop region ${ }^{7}$. However, we found that the residues most perturbed upon binding of $\mathbf{1}$ to ASH1L correspond to the amino acids surrounding the autoinhibitory loop (Fig. 2b). To confirm whether 1 binds to this region on ASH1L, we mutated S2259 to methionine to introduce steric hindrance in the autoinhibitory loop region (Supplementary Fig. 2b). Indeed, the NMR spectra of 
a

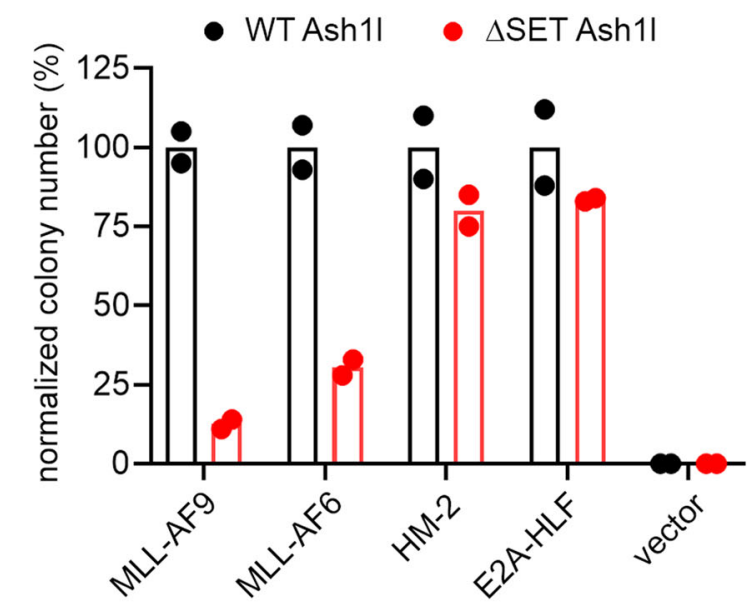

b

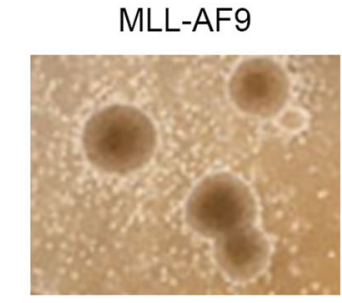

$\mathrm{HM}-2$

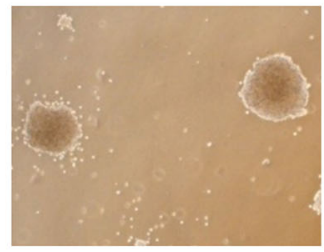

d
C

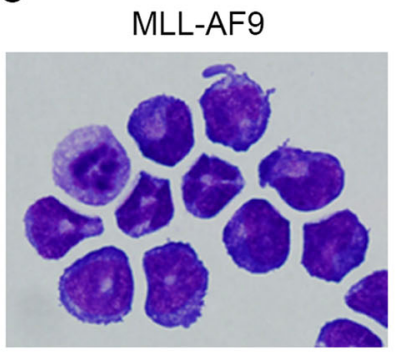

HM-2

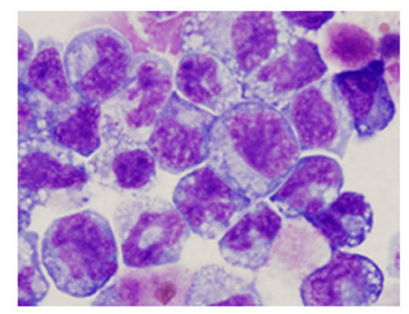

MLL-AF9 ( $\triangle$ SET Ash1I)

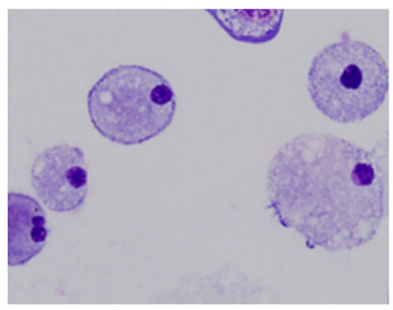

$\mathrm{HM}-2(\triangle \mathrm{SET}$ Ash1I)

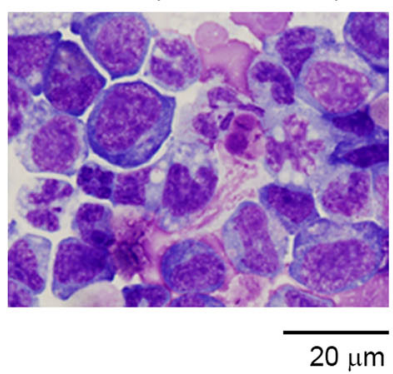

MLL-AF9 ( $\triangle$ SET Ash1l)

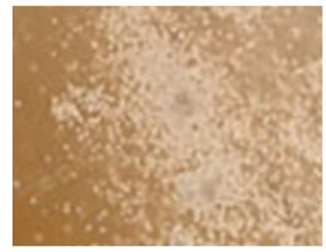

HM-2 ( $\triangle \mathrm{SET}$ Ash1l)
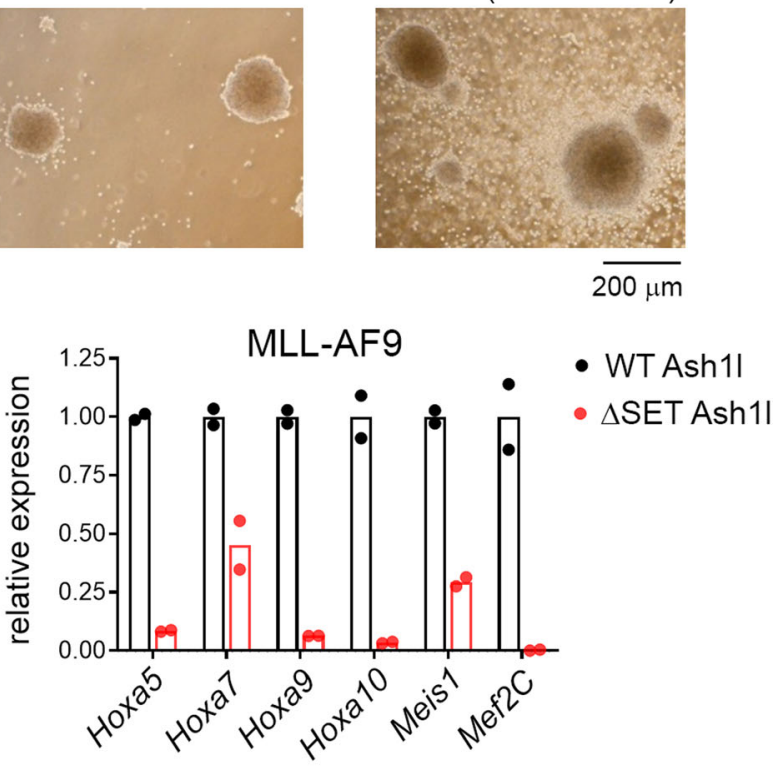

MLL-AF6

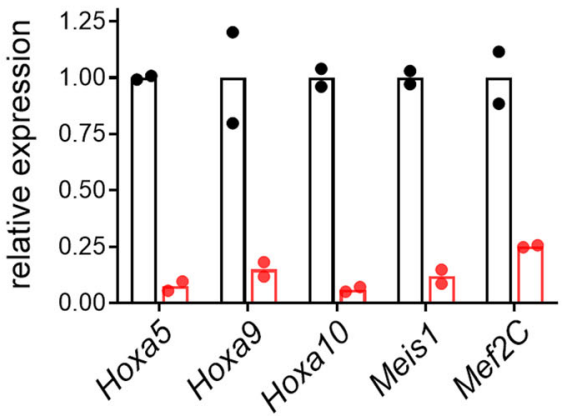

Fig. 1 SET domain of ASH1L is essential for efficient transformation by MLL fusion oncogenes. a Normalized colony counts from the round four of colony-forming assay performed in mouse bone marrow (BM) progenitor cells derived from the WT or $\triangle$ SET Ash1l mice and transduced with MLL-AF9, MLL-AF6, HOXA9/MEIS1 (HM-2), E2A-HLF or vector alone (MSCV). $n=2$. b, c Representative pictures of colonies (b) or Wright-Giemsa stained cytospins (c) from the round four of colony assay performed in bone marrow cells derived from WT or $\triangle$ SET Ash1l mice and transduced with MLL-AF9 or HOXA9/ MEIS1 (HM-2). The experiment was repeated twice with similar results. d Quantitative RT-PCR performed in BM cells derived from WT or $\triangle$ SET Ash1l mice and transduced with MLL-AF9 or MLL-AF6. Gene expression was normalized to Gapdh and gene expression changes in $\Delta$ SET Ash1l cells were referenced to the corresponding values in the WT Ash1l background. Data represent two independent experiments each performed in duplicates.

the ASH1L S2259M showed no chemical shift perturbations in the presence of $\mathbf{1}$, validating no binding and supporting that our fragment hit binds to the autoinhibitory loop region (Supplementary Fig. 2b,c).

Chemical optimization of a fragment hit 1 . To improve the potency of $\mathbf{1}$, we undertook medicinal chemistry optimization and found that the replacement of the pyrrole with an indole, which resulted in compound $\mathbf{2}$ (Fig. $2 \mathrm{c}$ ), substantially improved binding affinity, as evidenced by more extensive chemical shift perturbations on the NMR spectra (Fig. 2d). To test whether 2 inhibits catalytic activity of ASH1L, we used an in vitro enzymatic assay with nucleosomes as the substrate ${ }^{7}$ and found that 2 blocked
ASH1L activity with an $\mathrm{IC}_{50}$ value of $50 \mu \mathrm{M}$ (Fig. 2c,e). Interestingly, despite the moderate inhibitory activity, we observed slow exchange kinetics on the ${ }^{15} \mathrm{~N}-{ }^{1} \mathrm{H}$ TROSY-HSQC spectra upon binding of 2 to the SET domain of ASH1L, indicating a slow offrate for the dissociation of the inhibitor from the protein (Fig. 2d).

Next, we substituted the indole nitrogen in compound $\mathbf{2}$ with a hydroxyethyl group, yielding compound 3 with three-fold improved inhibitory activity $\left(\mathrm{IC}_{50}=15 \mu \mathrm{M}\right.$, Fig. $\left.2 \mathrm{c}, \mathrm{e}\right)$. In addition, the substitution of the indole at position six with the aminomethyl group resulted in $\mathbf{4}$ with a two-fold improved inhibitory activity $\left(\mathrm{IC}_{50}=23 \mu \mathrm{M}\right)$ over 2 . By combining both substituents we synthesized compound 5 (AS-5), which exhibited low micromolar activity $\left(\mathrm{IC}_{50}=4.0 \mu \mathrm{M}\right.$, Fig. $2 \mathrm{c}$, e). We then used isothermal 
a

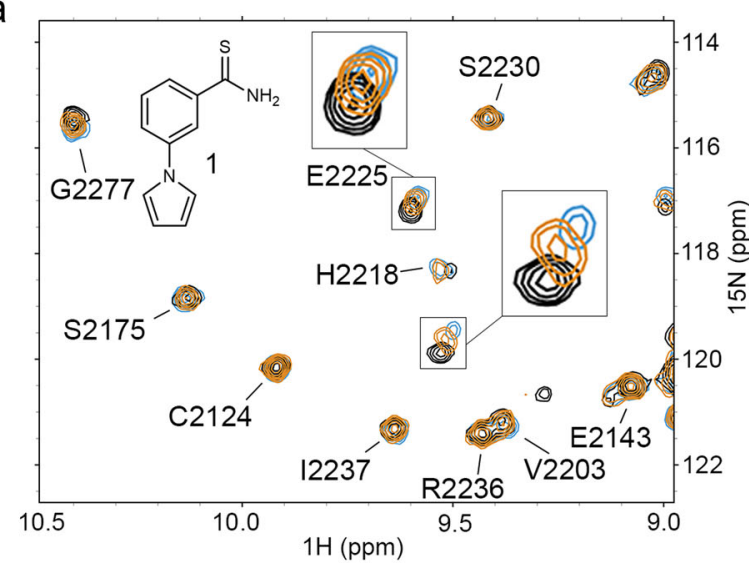

C

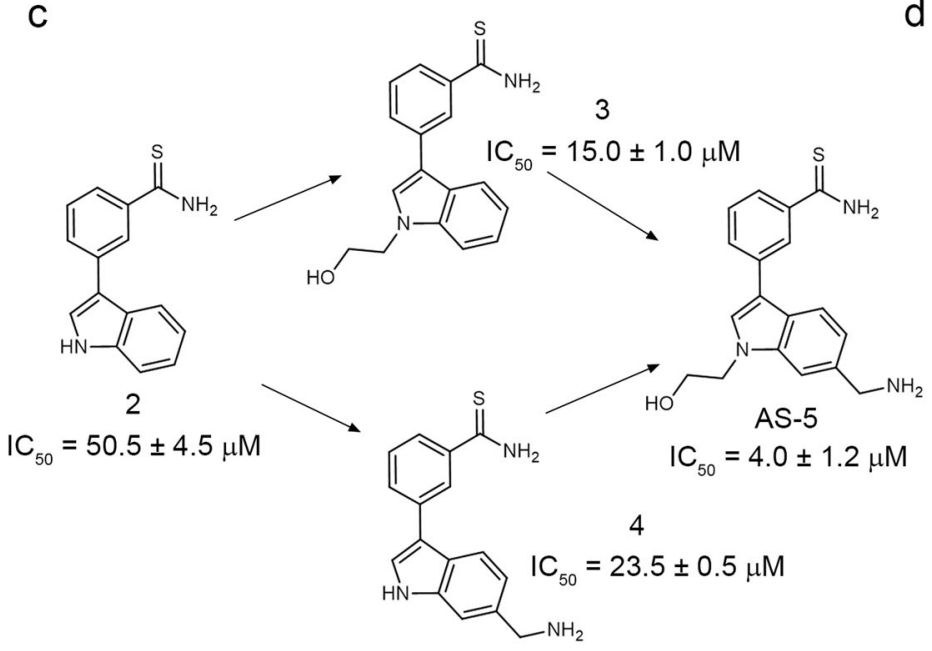

e

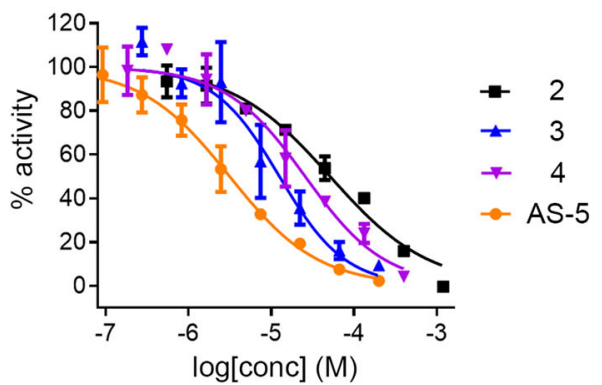

b

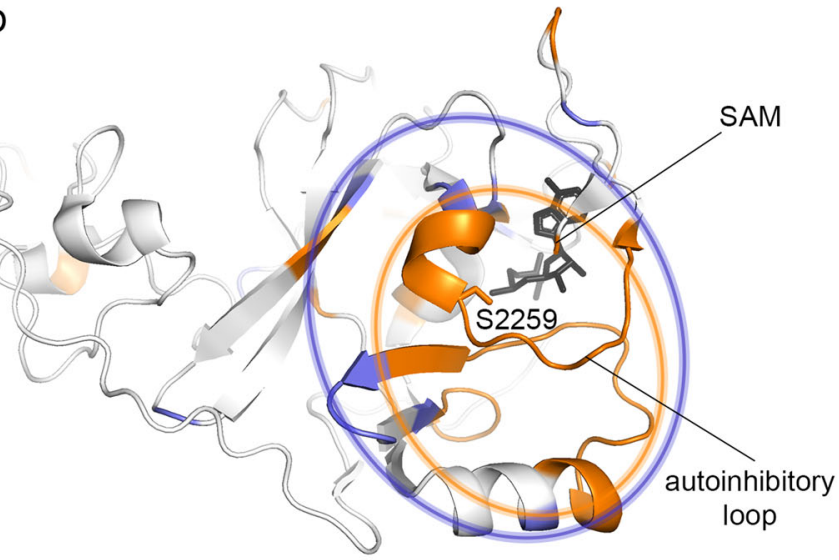

d

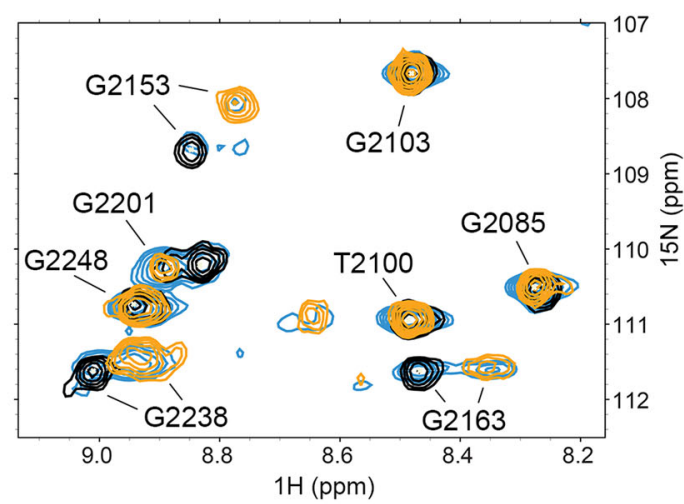

\section{f}

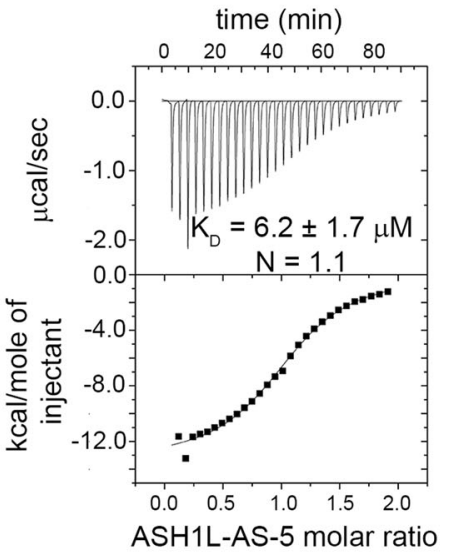

Fig. 2 Development and characterization of ASH1L inhibitors. a Structure of the fragment hit, compound $\mathbf{1}$, and its binding to ASH1L SET domain. Superposition of the ${ }^{1} \mathrm{H}-{ }^{15} \mathrm{~N}$ TROSY-HSQC spectra of $100 \mu \mathrm{M}$ ASH1L with $5 \%$ DMSO (black) with $500 \mu \mathrm{M}$ (orange) or $1 \mathrm{mM}$ (blue) of compound 1. b Chemical shift perturbations upon binding of 1 to ASH1L mapped on the crystal structure of ASH1L SET domain (PDB code 4YNM). Residues experiencing chemical shift perturbations calculated as $\triangle_{\mathrm{HN}}=\sqrt{\left(\delta_{\mathrm{HN}}^{2}+\delta_{\mathrm{N}}^{2}\right)}$ larger than $15 \mathrm{~Hz}$ are colored and encircled in violet. Residues unobserved or unassigned are colored and encircled in orange. c Chemical structures and activities for selected ASH1L inhibitors. IC 50 values represent mean \pm s.d. from two independent experiments. d Superposition of the ${ }^{1} \mathrm{H}_{-}{ }^{15} \mathrm{~N}$ TROSY-HSQC spectra of $100 \mu \mathrm{M}$ ASH1L with $5 \%$ DMSO (black) and with $500 \mu \mathrm{M}$ (orange) or $100 \mu \mathrm{M}$ (blue) of AS-5. e Titration curves from the HMT assay for ASH1L with compounds presented in panel (c) mean \pm s.d. Representative curves are shown from two independent experiments, each performed in duplicates. $\mathbf{f}$ Binding isotherm from the ITC experiment performed for the binding of AS-5 to ASH1L. Data are mean \pm s.d. from two independent experiments. A representative binding isotherm is shown. $\mathrm{N}$ represents stoichiometry of binding.

titration calorimetry (ITC) to quantify the binding affinity of AS-5 to ASH1L SET and obtained 1:1 stoichiometry and $\mathrm{K}_{\mathrm{d}}$ of $6.2 \mu \mathrm{M}$, which is consistent with the inhibitory activity for this compound (Fig. 2f). Overall, these efforts led to over 200-fold improvement of the activity for AS-5 versus the original fragment hit.

AS-5 binds to the autoinhibitory loop region of ASH1L SET. To further characterize the binding of ASH1L inhibitors we crystallized the ASH1L SET domain in complex with AS-5. Crystals of the wild-type SET domain diffracted to a low resolution $(\sim 3 \AA)$, precluding detailed structural analysis. In contrast, co-crystallization of AS-5 with ASH1L SET Q2265A mutant ${ }^{7}$ yielded well diffracting crystals (1.69 $\AA$ resolution), enabling structure determination of the complex, which showed a well-defined electron density map of AS-5 bound to ASH1L (Fig. 3a, Supplementary Fig. 3a and Supplementary Table 1). The ASH1L-AS-5 structure revealed that the compound binds to a predominantly buried site formed by residues from the 

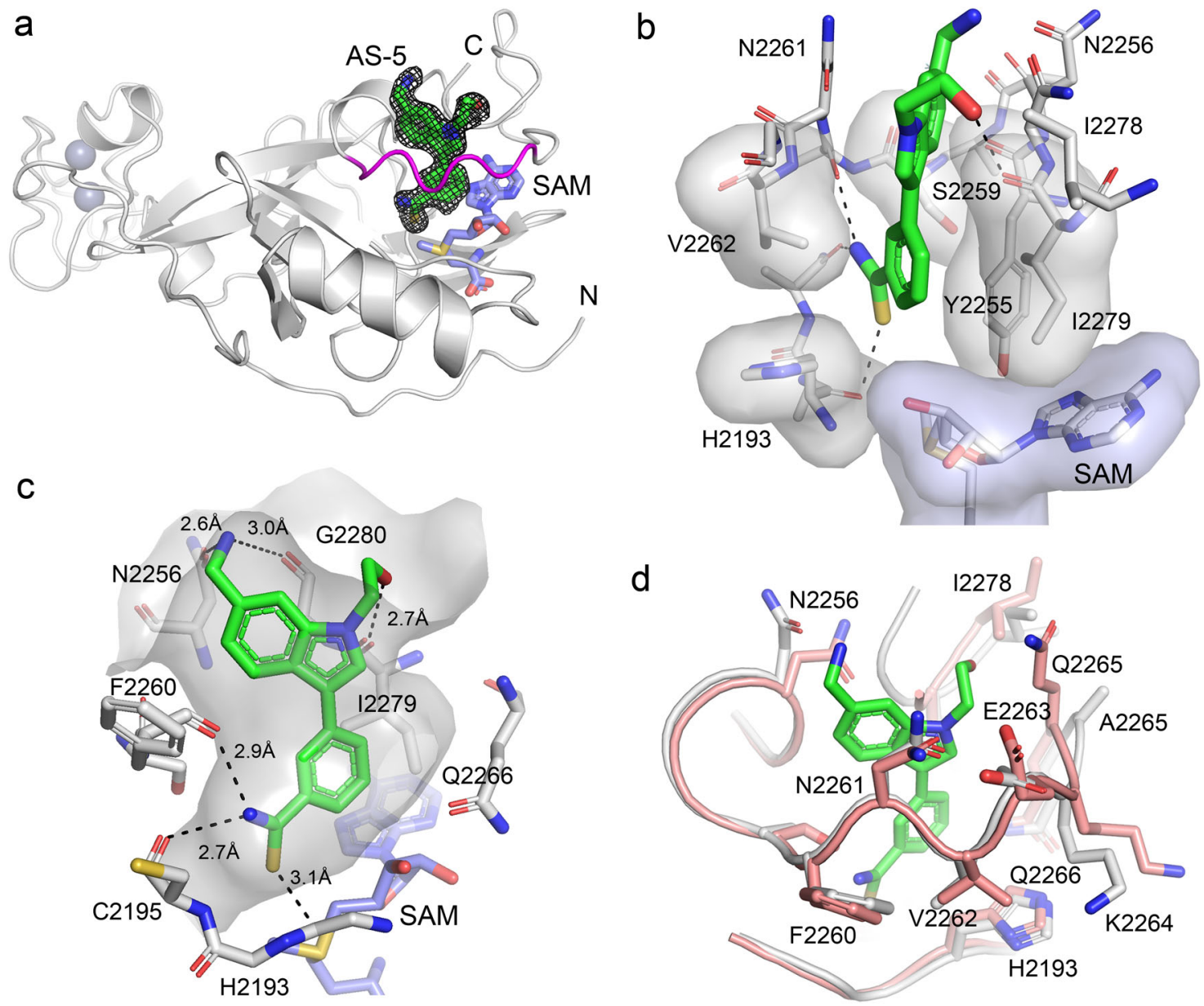

Fig. 3 Crystal structure of ASH1L SET in complex with AS-5. a Overall structure of ASH1L-AS-5 complex with 2Fo-Fc electron density map for AS-5 contoured at the $1 \sigma$ level. Protein is shown as ribbon with autoinhibitory loop in magenta and AS-5 shown in sticks with green carbons. b ASH1L residues involved in hydrophobic contacts with AS-5 shown in sticks and transparent surfaces (gray) and SAM is shown in sticks with the blue surface. Hydrogen bonds and chalcogen bonds are shown as dashed lines. Color-coding: AS-5 carbons (green), protein and SAM carbons (gray), oxygens (red), nitrogen (blue), sulfur (yellow). c Details of internal pocket in ASH1L (shown as semi-transparent surface) and binding mode of AS-5 (shown in sticks with green carbons). Selected residues in the ASH1L binding site are shown as sticks with color coding is as in panel (c). Hydrogen bonds and chalcogen bonds are shown as dashed lines with distances in $\AA$. $\mathbf{d}$ Superposition of ASH1L structure (PDB code 4YNM with salmon carbons) on the structure of ASH1L-AS-5 complex (protein with gray carbons and AS-5 with green carbons).

autoinhibitory loop and the C-terminus of the SET domain and is restricted at the bottom by the SAM co-factor (Fig. 3a). This site is lined by the hydrophobic portions of the side chains of H2193, Y2255, S2259, V2262, and I2279, the polar groups from the side chains of N2256, N2261, and Q2266, and the SAM co-factor (Fig. 3bd). An interesting feature of the binding site on ASH1L is the presence of multiple backbone carbonyls from S2259, C2159, H2193, N2256, F2260, K2264, and G2280, most of which are involved in the interactions with AS-5 (Fig. 3b, c). Overall, the crystal structure shows high shape complementarity of AS-5 to the binding site in the ASH1L SET domain (Fig. 3b, c). The thioamide group of AS-5 is deeply inserted into the pocket in ASH1L and is engaged in a network of high-quality interactions, including two hydrogen bonds with carbonyl groups of C2195 and F2260, Fig. 3b, c. In addition, the thioamide group is located at a close distance $(\mathrm{O} \cdots \mathrm{S}=\mathrm{C}$ distance of $3.1 \AA)$ and has favorable geometry $\left(\mathrm{O} \cdots \mathrm{S}=\mathrm{C}\right.$ angle of $\left.146^{\circ}\right)$ to the carbonyl group of $\mathrm{H} 2193$, suggesting the formation of the chalcogen bond $^{20}$ (Fig. 3b, c). The hydroxyethyl substituent on the indole nitrogen forms a hydrogen bond with the backbone carbonyl of I2279, while the aminomethyl group is involved in hydrogen bonds with the backbone carbonyl of G2280 and with the side chain of N2256 (Fig. 3b, c).
We have previously reported that the autoinhibitory loop in ASH1L experiences conformational dynamics that regulate its enzymatic activity ${ }^{7}$. The crystal structure of the ASH1L SET domain in the absence of the ligand reveals the presence of a relatively narrow crevice between the autoinhibitory and Cterminal loops, with the autoinhibitory loop blocking access to the active site. The binding of AS-5 to this region induces relatively small conformational changes (Fig. 3d and Supplementary Fig. 3b) and suggests that AS-5 exploits conformational heterogeneity in the ASH1L SET domain rather than inducing large conformational changes upon binding to the protein.

Structure-based design and development of potent ASH1L inhibitors. Analysis of the crystal structure of the ASH1L-AS-5 complex suggests that the protein could accommodate larger substituents at the indole nitrogen and showed that the aminomethyl group in AS-5 points towards the C-terminus of an ahelix in the SET domain. Thus, we replaced the hydroxyethyl group at indole nitrogen of AS-5 with 1-(methylsulfonyl)piperidine substituent and introduced a positively charged imidazole moiety at position six of indole to afford favorable interactions 
a<smiles>CS(=O)(=O)N1CCC(n2cc(-c3cccc(C(N)=O)c3)c3ccc(CNC(=O)Cc4c[nH]cn4)cc32)CC1</smiles><smiles>CN1CC(C(=O)NCc2ccc3c(-c4cccc(C(N)=O)c4)cn(C4CCN(S(=O)(=O)C(F)(F)F)CC4)c3c2)CN1C(=O)NCc1ccc2c(-c3cccc(C(N)=S)c3)cn(C3CCN(S(=O)(=O)F)CC3)c2c1</smiles>

b
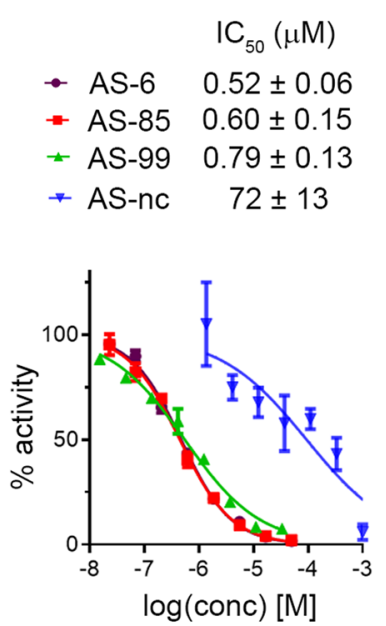

C

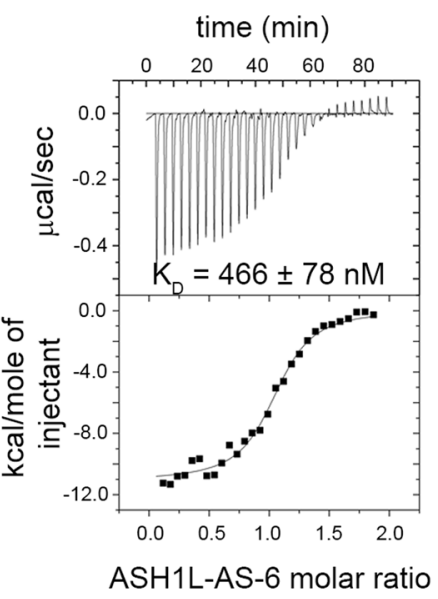

d

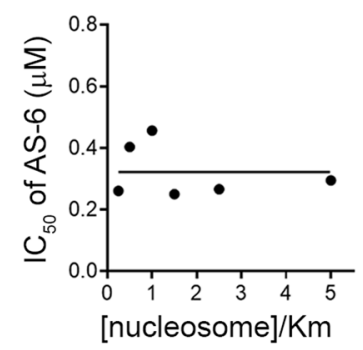

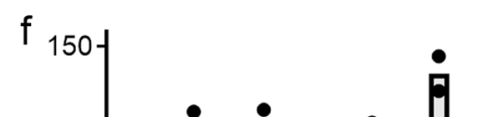

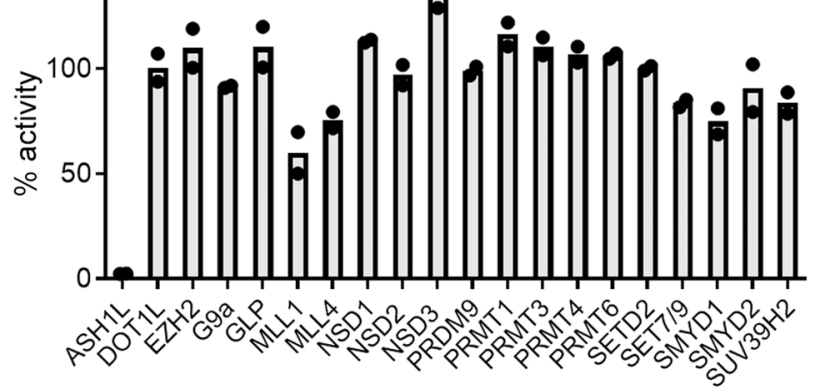

e

G2281

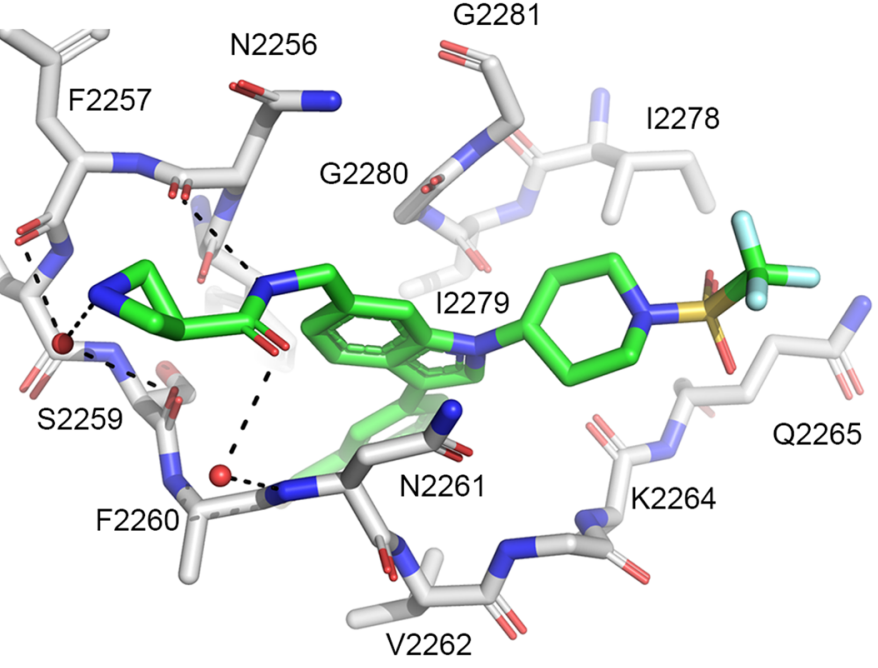

Fig. 4 Structure-based optimization of ASH1L inhibitors. a Chemical structures of ASH1L inhibitors developed using structure-based design. $\mathbf{b}$ Titration curves and $\mathrm{IC}_{50}$ values from the HMT assay. Data are mean \pm s.d. from two independent experiments. $\mathbf{c}$ Binding isotherm from the ITC experiment performed for the binding of AS-6 to ASH1L. Data are mean \pm s.d. from two independent experiments. A representative binding isotherm is shown. d Mechanistic studies from HMT assay with the $\mathrm{IC}_{50}$ values for AS-6 measured at various nucleosome concentration demonstrating non-competitive inhibition with nucleosome. e Crystal structure of ASH1L-AS-85 complex determined at $1.69 \AA$ resolution. Selected ASH1L residues (gray carbons) and AS85 (green carbons) are shown in sticks. Hydrogen bonds are shown as dashed lines. $\mathbf{f}$ Selectivity of AS-99 tested at $50 \mu \mathrm{M}$ concentration against a panel of histone methyltransferases. Data represent two independent experiments each performed in duplicates.

with the helix dipole, resulting in compound 6 (AS-6), (Fig. 4a). Importantly, compound AS-6 demonstrated $\sim 10$-fold improved inhibitory activity over AS-5, resulting in an $\mathrm{IC}_{50}$ value of 0.52 $\mu \mathrm{M}$ and $\mathrm{K}_{\mathrm{d}}$ of $466 \mathrm{nM}$ for binding to ASH1L as measured by ITC (Fig. 4b, c).

The binding mode of the ASH1L inhibitors that we developed suggests a requirement for the SAM co-factor for their effective binding (Fig. 3a-c). To further investigate the mechanism of inhibition and evaluate whether AS-6 is competitive with the nucleosome substrate, we tested the activity of AS-6 as a function of nucleosome concentration. Interestingly, AS-6 demonstrates the ASH1L inhibition that is non-competitive with the nucleosome, suggesting that the inhibitor does not directly prevent substrate binding to the SET domain (Fig. 4d). Such a model remains in agreement with the reports emphasizing the requirement for ASH1L motifs outside of the SET domain to achieve catalytic activity, likely by facilitating nucleosome binding 7,21 .

Despite potent in vitro activity, AS-6 demonstrated a very limited effect on the proliferation of the MLL leukemia cells $\left(\mathrm{GI}_{50} \sim 50 \mu \mathrm{M}\right)$ (Supplementary Fig. 4), possibly due to poor cell permeability associated with the higher polarity of this compound $(\operatorname{cog} \mathrm{P}=1.6$, tPSA $=120$ ), Supplementary Table 2. Thus, we designed two further analogs of AS-6 by introducing 1-(trifluoromethylsulfonyl) piperidine moiety at the indole nitrogen and azetidine or methylazetidine rings at position six of the indole, yielding AS-85 $(\operatorname{cog} \mathrm{P}=3.4, \mathrm{tPSA}=108)$ and AS-99 $(\operatorname{clog} \mathrm{P}=4.1, \mathrm{tPSA}=99)$, respectively (Fig. 4a). Importantly, both compounds are potent 
ASH1L inhibitors $\left(\mathrm{IC}_{50}\right.$ of 0.60 and $0.79 \mu \mathrm{M}$ for AS-85 and AS-99, respectively), and strongly bind to the ASH1L SET domain, with the $\mathrm{K}_{\mathrm{d}}$ values of 0.78 and $0.89 \mu \mathrm{M}$, respectively, Fig. $4 \mathrm{a}, \mathrm{b}$ and Supplementary Fig. 5a. Both analogs, AS-85 and AS-99 have been designed to increase $\operatorname{cLogP}$ and decrease tPSA when compared to AS-6, with the aim to improve their cell permeability. We also noticed that ligand efficiency (LE) ${ }^{22}$ for these compounds slightly dropped during the lead optimization process (from $\sim 0.32$ to $\sim 0.24$ ), which was associated with the need to improve the activity, cell permeability and drug-like properties, Supplementary Table 2.

To understand how optimized inhibitors bind to ASH1L, we determined the crystal structure of the wild-type ASH1L in complex with AS-85 (1.69 Å resolution) and found well defined electron density of the ligand (Fig. 4e and Supplementary Fig. 5b). Overall, the binding mode of $\mathbf{A S - 8 5}$ is very similar to AS-5, and the common core structure occupies the same site on ASH1L (Fig. 3 and Fig. 4e). The piperidine moiety at indole nitrogen fits into a ledge formed by the side chains of E2263, Q2265, and I2278 as well as the backbone of the C-terminal residues G2280 and G2281 (Fig. 4e). The positively charged azetidine ring of AS85 forms favorable contacts with the helix dipole from the short helical fragment encompassing residues from N2254 to H2258 and a water-mediated hydrogen bond with F2257 (Fig. 4e). In addition, the amide group in the linker extending from the indole ring at position six is involved in a hydrogen bond with the backbone carbonyl of N2256 and a water-mediated hydrogen bond with N2261 (Fig. 4e).

An intriguing feature of this class of ASH1L inhibitors is the presence of a thioamide group, which forms a unique network of interactions, including the chalcogen bond between the sulfur in thioamide and the backbone carbonyl of H2193 (Fig. 3b and Supplementary Fig. 5b). To assess whether sulfur in the thioamide is required for high binding affinity we replaced the thioamide in AS-99 with an amide group, yielding AS-nc (Fig. 4a). Surprisingly, the replacement of sulfur with oxygen resulted in $\sim 100$-fold reduced inhibitory activity, yielding an $\mathrm{IC}_{50}$ of $72 \mu \mathrm{M}$ for AS-nc (Fig. 4b), emphasizing the high relevance of the chalcogen bond involving the thioamide group in this class of ASH1L inhibitors. The modeling demonstrates a relatively close distance between the amide oxygen in AS-nc and the carbonyl group of $\mathrm{H} 2193$, which likely results in repulsive interactions, significantly lowering the activity of AS-nc versus AS-99 (Supplementary Fig. 5c). Since AS-nc has poor in vitro activity and a very similar structure to AS-99, we used this compound as a negative control for cellbased studies.

Structural analysis revealed that the ASH1L inhibitors reported here bind to the pocket formed by the autoinhibitory loop representing a characteristic feature of H3K36 histone methyltransferases, including the NSD family and SETD $2^{23}$. To evaluate the selectivity of these compounds towards ASH1L, we tested AS99 against a panel of 20 histone methyltransferases, including NSD1, NSD2, NSD3, and SETD2. Importantly, no significant inhibition was observed at $50 \mu \mathrm{M}$ of AS-99 on any of the tested HMTs, indicating over 100 -fold selectivity towards ASH1L (Fig. 4f). Furthermore, AS-99 did not show substantial inhibition in a panel of representative kinases (Supplementary Fig. 6). Overall, AS-99 is a sub-micromolar ASH1L inhibitor with a favorable selectivity profile and no substantial off-target effects.

AS-99 shows on-target activity in MLL leukemia cells. Based on our findings demonstrating the importance of the ASH1L SET domain in MLL leukemia (Fig. 1), we evaluated the effect of the ASH1L inhibitors in a panel of leukemia cells with MLL1 translocations. First, we found that AS-85 inhibits the growth of leukemia cells (MV4;11, MOLM13, and KOPN8) harboring different
MLL1 translocations, with $\mathrm{GI}_{50}$ values ranging from $5 \mu \mathrm{M}$ to 25 $\mu \mathrm{M}$, and demonstrates no effect in K562 leukemia cells without MLL1 translocations (Supplementary Fig. 7a,b). We also observed time-dependent cell growth inhibition for AS-85, a characteristic feature of histone-methyltransferase inhibitors ${ }^{24,25}$, with 7-10 days representing an optimal treatment time (Supplementary Fig. 7a). Furthermore, a more pronounced effect on the growth of the MLL leukemia cells was observed for AS-99, with the $\mathrm{GI}_{50}$ values ranging from $1.8 \mu \mathrm{M}$ to $3.6 \mu \mathrm{M}$ (Fig. 5a), likely due to the improved cell permeability over AS-85 resulting from the methyl substitution of the basic nitrogen in the azetidine ring (Fig. 4a), which led to higher cLogP and decreased tPSA (Supplementary Table 2). Importantly, AS-99 showed a several fold weaker effect on the proliferation of leukemia cells without MLL1 translocations, such as SET2 and K562, with no or limited effects at $10 \mu \mathrm{M}$ or higher concentrations (Fig. 5a). Finally, we also tested the structurally related AS-nc (serving as a negative control) in MLL leukemia cells and found no or much weaker effects (Fig. 5b). To assess potential toxicity in normal cells, we tested AS-99 in normal hematopoietic progenitor cells (human CD34+ cells from cord blood) and found no effect on colony number or morphology (Fig. 5c).

ASH1L knockdown ${ }^{9}$ and deletion of the ASH1L SET domain (Fig. 1) induced differentiation and apoptosis of MLL leukemia cells. Similarly, pharmacologic inhibition of ASH1L with AS-99, but not AS-nc, induced differentiation in MLL leukemia cells (MV4;11 and KOPN8) as assessed by markedly (up to 50\%) increased expression of the CD11B differentiation marker and cell morphology changes (Fig. 5d, e and Supplementary Fig. 8a). These effects were not observed in the control leukemia cell line K562, thus supporting the on-target mechanism of action of AS99 (Fig. 5d, e). In addition, AS-99 also induced apoptosis in the MLL leukemia cells, but not in the K562 cells, as assessed by the quantification of the Annexin V positive cells (Fig. 5f and Supplementary Fig. 8b). The effects on differentiation or apoptosis were not observed upon treatment of MLL leukemia cells with AS-nc (Supplementary Fig. 9a-c), further supporting the on-target activity of AS-99.

To assess whether AS-99 blocks H3K36 methylation in cells, we performed a CUT\&RUN experiment in MV4;11 cells. Indeed, treatment with AS-99 resulted in a reduced number of H3K36me2 peaks when compared to the DMSO-treated cells, confirming that AS-99 blocks the enzymatic activity of ASH1L in the MLL1-rearranged leukemia cells (Fig. 5g). Overall, these results further support the on-target activity of AS-99 in leukemia cells.

AS-99 suppresses MLL fusion driven transcriptional programs. To evaluate the mechanism of action of the ASH1L inhibitors, we performed gene expression studies with AS-99 and AS-nc in MOLM13 and MV4;11 MLL leukemia cells harboring MLL-AF9 and $M L L-A F 4$ translocations, respectively. The treatment of these cells with AS-99 led to a dose-dependent downregulation of canonical MLL fusion target genes required for leukemogenesis $9,19,26$, including MEF2C, DLX2, FLT3, and HOXA9 (Fig. 6a, b). In contrast, no changes in the expression of these genes were observed for the negative control compound ASnc, (Supplementary Fig. 10a, b). These effects were accompanied by a substantial increase in the expression level of the MNDA differentiation marker upon treatment with AS-99, but not with AS-nc, (Fig. 6a, b and Supplementary Fig. 10a, b), which is consistent with the differentiating phenotype of these cells (Fig. 5e).

Next, we performed global gene expression studies using RNAseq after seven days of treatment of MV4;11 cells with AS-99 and observed more downregulated (323) than up-regulated (75) genes 
a

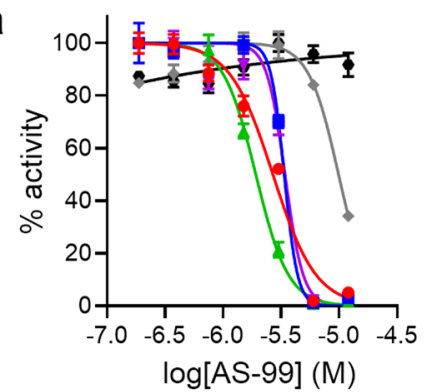

C

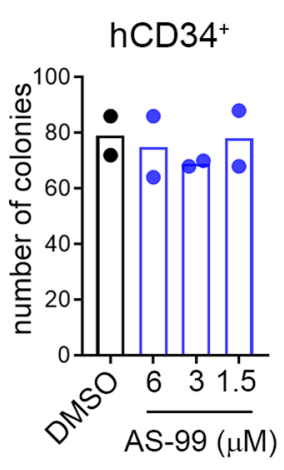

$\mathrm{GI}_{50}(\mu \mathrm{M})$

$\rightarrow$ KOPN8 $2.9 \pm 0.3$

- MOLM13 $3.6 \pm 0.3$

\# MV4:11 $1.8 \pm 0.2$

* RS4;11 $3.4 \pm 0.1$

$\rightarrow$ K562

$\rightarrow$ SET2

$\sim 10$
$>12$

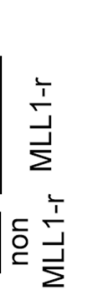

b

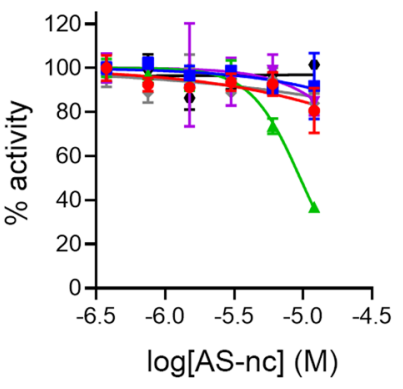

- KOPN8

- MOLM13

- MV4;11

- RS4;11

$\rightarrow \mathrm{K} 562$

- SET2

e
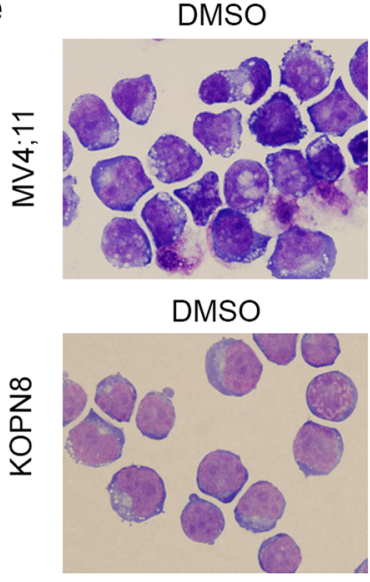

DMSO

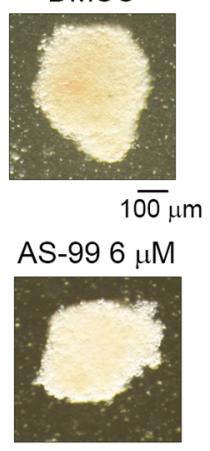

d $\quad$ MV4;11

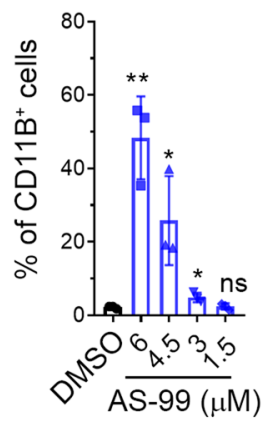

KOPN8

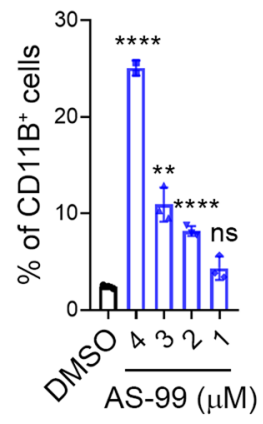

K562

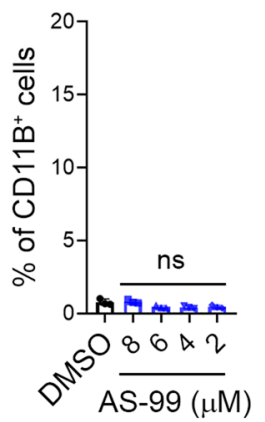

g
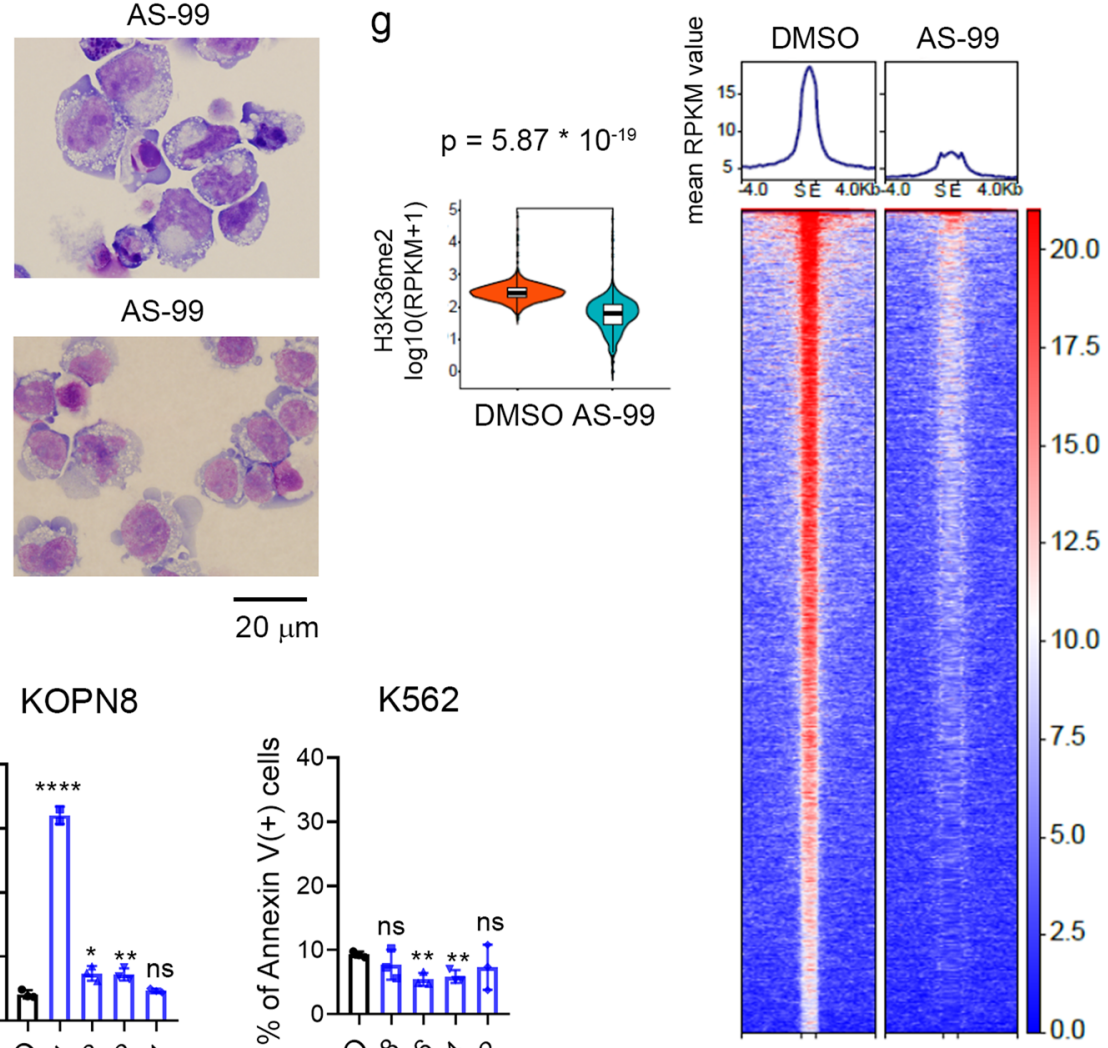

f

MV4;11

KOPN8

K562
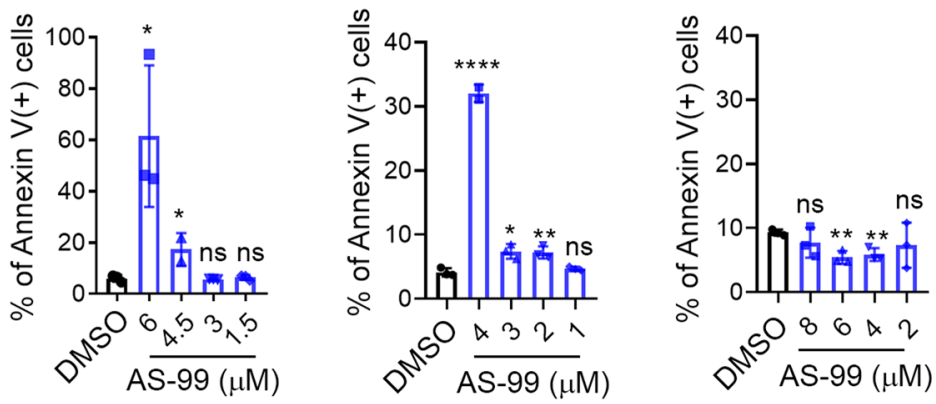

20.0

17.5

5.0

12.5

10.0

7.5

5.0

2.5
(Fig. 6c). This was found to be consistent with reduced H3K36me2 level (Fig. 5g), which is associated with active transcription $^{2}$. Importantly, Gene Set Enrichment Analysis (GSEA) revealed the downregulation of transcriptional programs driven by MLL fusion proteins and HOXA9 upon treatment of MV4;11 cells with AS-99. For example, AS-99 led to the downregulation of genes induced by the expression of
MLL-AF9 (Fig. 6d) ${ }^{27}$. Furthermore, AS-99 resulted in the suppression of the genes upregulated by NUP98-HOXA9 oncogene $^{28}$, suggesting that our ASH1L inhibitor represses the HOXA9-regulated program (Fig. 6e). All of these results strongly support that the inhibition of ASH1L catalytic activity with a small molecule inhibitor reverses the leukemogenic activity of MLL fusion proteins. 
Fig. 5 Cellular activity of ASH1L inhibitor AS-99. a, b Titration curves from the MTT cell viability assay performed after 7 days of treatment of human MLL1 rearranged (MLL1-r) leukemia cell lines (MV4;11, MOLM13, KOPN8, RS4;11) and control leukemia cell lines, non-MLL1-r (K562 and SET2) with AS-99 (a) or AS-nc (b); mean $\pm \mathrm{SD}, n=4$ biological replicates. Representative graphs are shown from 2-3 independent experiments. $\mathrm{GI}_{50}$ values correspond to AS-99 concentrations required to achieve 50\% inhibition of cell proliferation. c Colony counts and representative images of colonies from the colonyforming assay performed with normal human hematopoietic $\mathrm{CD}_{4}{ }^{+}$cells isolated from cord blood treated for 7 days with DMSO or AS-99; mean $\pm \mathrm{SD}$, $n=2$. The experiment was performed twice. Representative data is shown. $\mathbf{d}$ Quantification of CD11B expression in human leukemia cells treated for 7 days with AS-99, detected by flow cytometry; mean \pm SD, $n=3$ biological replicates. $P$ values (MV4;11: $6 \mu$ M: 0.0021, 4.5 $\mu$ M: 0.026, $3 \mu$ M: 0.028, 1.5 $\mu$ M: 0.73); KOPN8 ( $4 \mu \mathrm{M}<0.0001,3 \mu \mathrm{M}: 0.0012,2 \mu \mathrm{M}$ : <0.0001, $1 \mu \mathrm{M}$ : 0.055); K562 ( $8 \mu \mathrm{M}: 0.78,6 \mu \mathrm{M}: 0.070,4 \mu \mathrm{M}: 0.055,2 \mu \mathrm{M}: 0.081$ ) were calculated using unpaired 2-tailed $t$ test. Two independent experiments were performed for each cell line in triplicates. Representative graphs are shown. Gating strategy is presented in Supplementary Fig. 8c. e Wright-Giemsa-stained cytospins for MV4;11 and KOPN8 cells after 7 days of treatment with DMSO or AS-99: in MV4;11 at $6 \mu \mathrm{M}$ and in KOPN8 at $4 \mu \mathrm{M}$. f Flow cytometry analysis of apoptosis induced by AS-99 in MV4;11, KOPN8, and K562 cells after 7 days of treatment. Mean \pm SD, $n=3$ biological replicates. $P$ values (MV4;11: $6 \mu \mathrm{M}: 0.026,4.5 \mu \mathrm{M}: 0.049,3 \mu \mathrm{M}: 0.65,1.5 \mu \mathrm{M}: 0.70 ;$ KOPN8: $4 \mu \mathrm{M}:<0.0001,3$ $\mu \mathrm{M}: 0.012,2 \mu \mathrm{M}: 0.010,1 \mu \mathrm{M}: 0.24 ; \mathrm{K} 562: 8 \mu \mathrm{M}: 0.31,6 \mu \mathrm{M}: 0.004,4 \mu \mathrm{M}: 0.006,2 \mu \mathrm{M}: 0.39)$ were calculated using unpaired 2-tailed $t$ test. Two independent experiments were performed in triplicates. Representative graphs are shown. ${ }^{\star} P<0.05 ;{ }^{\star \star} P<0.01 ;{ }^{\star \star \star \star} P<0.0001 ; N S$ not significant. Gating strategy is presented in Supplementary Fig. 8d. g CUT\&RUN experiment in MV4;11 cells treated with AS-99 (5.5 $\mu$ M) or DMSO showing H3K36me2 peaks. The rows show the RPKM (Reads Per Kilobase Million) values on the peaks (normalized to $1 \mathrm{~kb}$ ) and $4 \mathrm{~kb}$ regions flanking the peaks. Peaks were sorted by total normalized signals of DMSO-treated cells within each category. Violin plots show the RPKM values of each peak in DMSO and AS-99 treated cells. The boxes represent 25th (minima) and 75th (maxima) percentile and the lines represent the median. The high and low whisker ends are the largest values within 1.5-times interquartile range above 75th and the smallest value within 1.5-times interquartile range below the 25th percentile, respectively. Mann-Whitney two-sided $U$ test was used for statistical analysis to compare the difference of signals between DMSO- and AS-99-treated cells.

AS-99 reduces leukemia burden in mice. To assess the utility of AS-99 for in vivo studies in mice we performed pharmacokinetic (PK) studies of this compound in mice, which revealed favorable exposure in plasma upon i.v. and i.p. administration (AUC $=$ $9701 \mathrm{hr}^{*} \mathrm{ng} / \mathrm{mL}$ and $10,699 \mathrm{hr}^{*} \mathrm{ng} / \mathrm{mL}$, respectively), suitable half-life $(\sim 5-6 \mathrm{~h})$ and $\mathrm{C}_{\max }>10 \mu \mathrm{M}$, Supplementary Fig. 11a,b. To evaluate the potential therapeutic effects of the ASH1L inhibitors, we performed an in vivo study with AS-99 using a xenotransplantation model of MLL leukemia with MV4;11 cells expressing luciferase transplanted into NSG mice ${ }^{29}$. The treatment with AS-99 (30 mg/kg, i.p., q.d.) or vehicle was initiated six days after transplantation and was continued for 14 consecutive days with the progression of the diseases monitored by bioluminescence signals (Fig. 6f). No substantial toxicity was observed during the treatment period as indicated by measurement of mice body weight, which was only slightly reduced (by $\sim 10 \%$ ) after 7 days of treatment with AS-99, but this difference became less pronounced as the treatment continued (Supplementary Fig. 11c). Importantly, the mice treated with AS-99 demonstrated a $65 \%$ reduction in the bioluminescence signal when compared to the vehicle-treated control mice (Fig. 6f, g). This effect was accompanied by a significantly lower level of leukemic blasts (human CD45 + cells) in the peripheral blood and spleen of the AS-99treated mice when compared to the vehicle-treated mice on the last day of the treatment (Fig. 6h). We have also assessed the effect of AS-99 in normal mice using the same treatment schedule as in the leukemia model and found no significant difference on blood counts and other blood parameters between AS-99 and vehicle-treated mice, Supplementary Fig. 12a. The T-cells were unaffected while myeloid and B-cells were slightly changed (increased and reduced, respectively) in the AS-99-treated mice, although they remained in the normal range, Supplementary Fig. 12b. Overall, AS-99 reduced the leukemia burden in the xenotransplantation mouse model of MLL leukemia without affecting blood counts in normal mice, indicating that this compound can serve as a valuable chemical probe and a candidate for further development.

\section{Discussion}

In this study, we validated the role of the catalytic ASH1L SET domain in MLL leukemia. Importantly, mice deficient with Ash $11^{30}$ or with a loss of the Ash1l SET domain ${ }^{5}$ remained viable with no major developmental defects, suggesting that targeting ASH1L may have limited or no side effects and supporting ASH1L as an attractive target for drug discovery. However, ASH1L is a challenging target for inhibitor development as the SET domain adapts an inactive conformation with the autoinhibitory loop blocking access to the active site 2,7 . By applying fragment-based screening, followed by medicinal chemistry and a structure-based design, we developed first-in-class and potent ASH1L inhibitors, including AS-99 $\left(\mathrm{IC}_{50}=0.79 \mu \mathrm{M}, \mathrm{K}_{\mathrm{d}}=0.89 \mu \mathrm{M}\right)$. The systematic application of biochemical, biophysical, and structural studies resulted in ASH1L inhibitors with over 1000-fold improved binding affinity over the initial fragment hit. Thus, our study reveals the tractability of the ASH1L SET domain for inhibition by small molecules, as no such compounds were reported before. One of the striking features of our ASH1L inhibitors essential for high potency is the presence of the thioamide group, as its replacement by an amide results in $\sim 100$-fold reduced inhibitory activity. This is likely due to the loss of a chalcogen bond involving sulfur from the thioamide group and electrostatic repulsion between the oxygen in the amide analog and the carbonyl group of $\mathrm{H} 2193$ (Supplementary Fig. 5c). This example emphasizes favorable interactions of sulfur in protein-ligand complexes.

Structural studies demonstrate that the inhibitors we developed bind to a site adjacent to the autoinhibitory loop in the SET domain, possibly stabilizing the conformation in this region and allosterically blocking the enzymatic activity of ASH1L. Interestingly, the autoinhibitory loop, which is present in only four other H3K36 histone methyltransferases (NSD1, NSD2, NSD3, and SETD2) ${ }^{2}$, has poorly conserved amino acid sequences, providing a rationale for the $>100$ fold selectivity of AS-99 to ASH1L over other HMTs.

When tested in MLL leukemia cells, AS-99 downregulated the expression of MLL fusion target genes and reduced H3K36 dimethylation, strongly supporting an on-target mechanism of action. The GSEA analysis revealed that AS-99 suppressed transcriptional programs driven by MLL fusion proteins and HOXA9. Treatment with AS-99 also inhibited proliferation and induced differentiation and apoptosis in MLL leukemia cells, but not in control cell lines without MLL1 translocation, excluding general toxicity. Thus, AS-99 recapitulates the effects observed upon genetic inactivation of $\mathrm{ASH}_{1} \mathrm{~L}^{9}$ or deletion of the ASH1L SET domain (this paper). When applied in vivo, AS-99 reduced the leukemia burden in a systemic model of MLL leukemia, supporting the inhibition of ASH1L as an approach to target leukemia with $M L L 1$ translocations. 
a

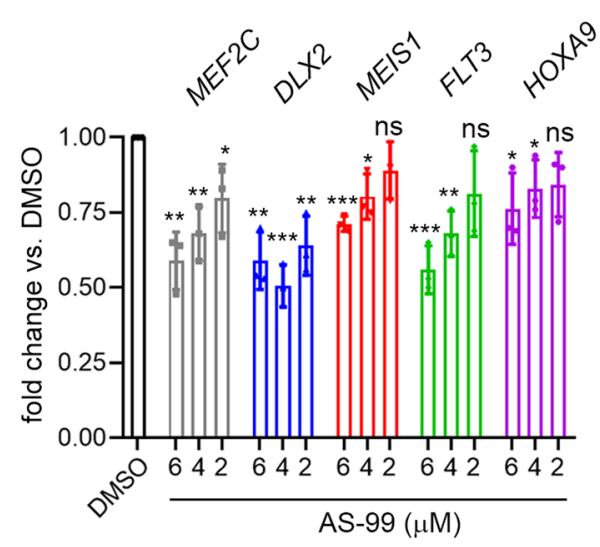

C

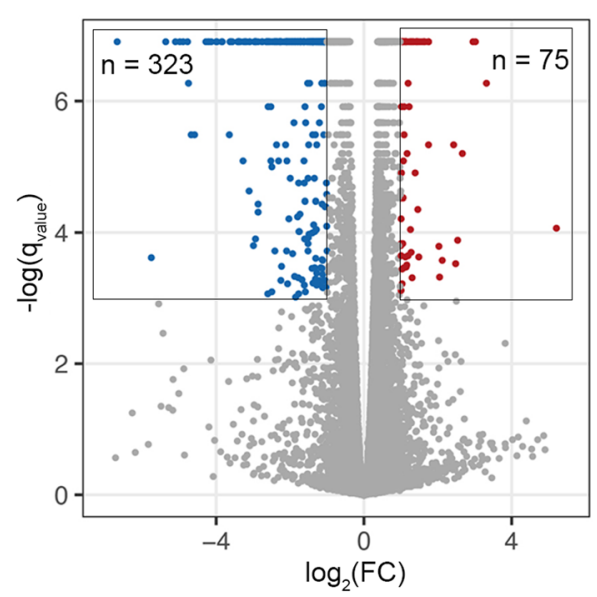

$f$

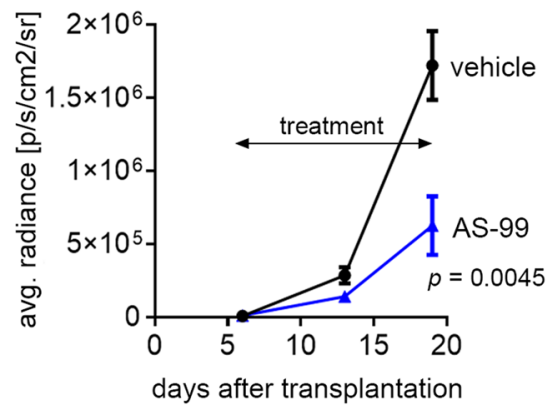

b

MV4;11

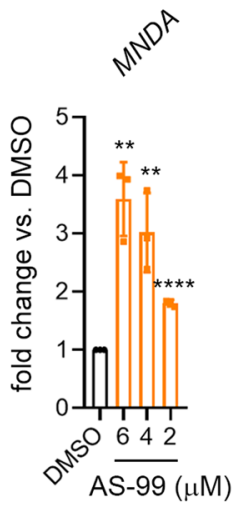

d

targets of MLL-AF9 (Kumar) $\mathrm{n}=280$ genes

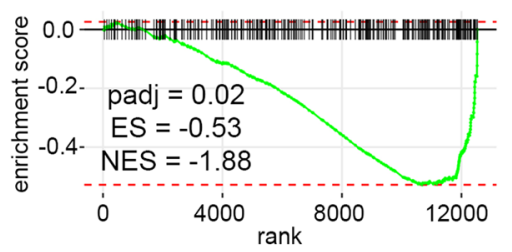

e

targets of NUP98-HOXA9 (Takeda) $n=112$

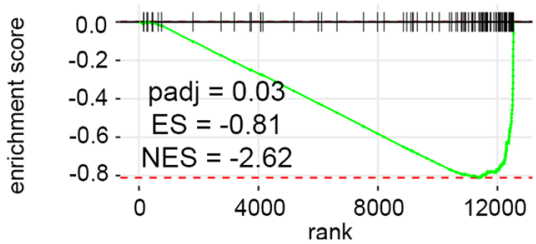

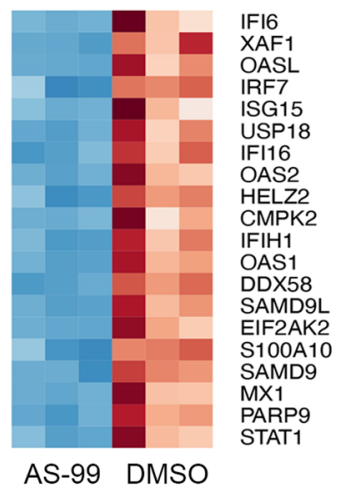

9

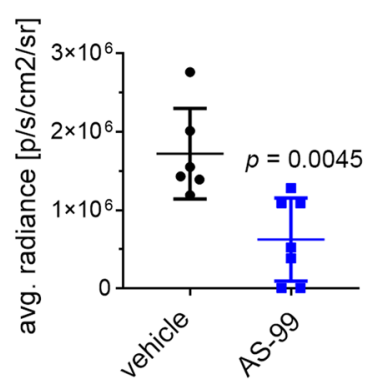

$\mathrm{h}$

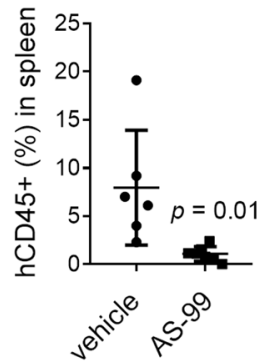

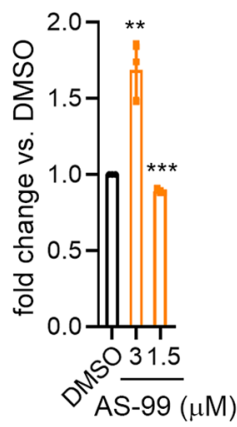

In summary, the AS-99 compound that we report here represents a first-in-class, well characterized, and selective ASH1L inhibitor with sub-micromolar in vitro activity. This compound can serve as a valuable chemical probe to further explore the biological functions of ASH1L and to address a potential therapeutic benefit of blocking ASH1L in different diseases, including cancer. The outcome of these studies will pave the way towards the development of pharmacological agents targeting the catalytic SET domain of ASH1L.

\section{Methods}

Protein expression and purification. ASH1L SET domain (amino acids 2069-2288) was expressed and purified as previously described ${ }^{7}$ and was used in
ITC, NMR, and X-ray crystallography experiments. Briefly, ASH1L (2069-2288) was fused with an $\mathrm{N}$-terminal Mocr tag ${ }^{31}$ and was expressed in E. Coli BL21(DE3) cells. Transformed cells were harvested and lysed, and the supernatant was loaded on a column packed with Ni-NTA agarose for affinity chromatography. The MocrASH1L fusion was eluted in the buffer containing $50 \mathrm{mM}$ Tris, $\mathrm{pH}=7.5,500 \mathrm{mM}$ $\mathrm{NaCl}, 1 \mathrm{mM}$ TCEP and $500 \mathrm{mM}$ imidazole, followed by the cleavage of Mocr tag using tobacco etch virus (TEV) protease. Cleaved ASH1L was isolated from Mocr by repeating the Ni-NTA affinity chromatography and collecting ASH1L eluted in the buffer containing $50 \mathrm{mM}$ Tris, $\mathrm{pH}=7.5,500 \mathrm{mM} \mathrm{NaCl}, 1 \mathrm{mM}$ TCEP and 20 $\mathrm{mM}$ of imidazole. For X-ray crystallography experiments, the protein was further purified by size-exclusion chromatography using a Superdex-75 column (GE Life Sciences). The ASH1L SET domain with Q2265A mutation (amino acids 20692288) was expressed and purified following the same protocol and was used in the X-ray crystallography experiments. An extended construct of the ASH1L SET 
Fig. 6 AS-99 impairs transcriptional program of MLL fusion proteins and reduces leukemia burden. $\mathbf{a}$, $\mathbf{b}$ Quantitative RT-PCR performed in MOLM13 cells (a) or MV4;11 cells (b) after 7 days of treatment with AS-99. Gene expression was normalized to HPRT1 and referenced to the DMSO treated cells. Representative data from two independent experiments, each performed in triplicates (mean $\pm S D, n=3$ biological replicates) are shown. $P$ values were calculated using unpaired 2-tailed $t$ test. For gene expression in MOLM13 cells, the $P$ values are as follows: MEF2C: $6 \mu M: 0.0017,4 \mu \mathrm{M}: 0.0035,2 \mu \mathrm{M}$ : 0.036, DLX2: $6 \mu \mathrm{M}$ 0.0017, $4 \mu \mathrm{M}: 0.0003,2 \mu \mathrm{M}: 0.0032$, MEIS1: $6 \mu \mathrm{M}:<0.0001,4 \mu \mathrm{M}: 0.011,2 \mu \mathrm{M}: 0.12 ;$ FLT3: $6 \mu \mathrm{M}: 0.0007,4 \mu \mathrm{M}: 0.0018,2 \mu \mathrm{M}: 0.086$; HOXA9: $6 \mu \mathrm{M}: 0.026,4 \mu \mathrm{M}: 0.038,2 \mu \mathrm{M}: 0.064 ;$ MNDA: $6 \mu \mathrm{M}: 0.0021,4 \mu \mathrm{M}: 0.0068,2 \mu \mathrm{M}:<0.0001$. For gene expression in MV4;11 cells, the $P$ values are as follows: MEF2C: $3 \mu \mathrm{M}: 0.0007,1.5 \mu \mathrm{M}: 0.009$, DLX2: $3 \mu \mathrm{M}:<0.0001,1.5 \mu \mathrm{M}: 0.0017$, MEIS1: $3 \mu \mathrm{M}: 0.16,1.5 \mu \mathrm{M}: 0.074$, FLT3: $3 \mu \mathrm{M}: 0.0010,1.5 \mu \mathrm{M}$ : 0.57, HOXA9: $3 \mu \mathrm{M}: 0.010,1.5 \mu \mathrm{M}: 0.044$, MNDA: $3 \mu \mathrm{M}: 0.0031,1.5 \mu \mathrm{M}: 0.0004 .{ }^{\star} P<0.05$; ${ }^{\star \star} P<0.01 ;{ }^{\star \star \star} P<0.001$; ${ }^{\star \star \star \star} P<0.0001$; NS not significant.

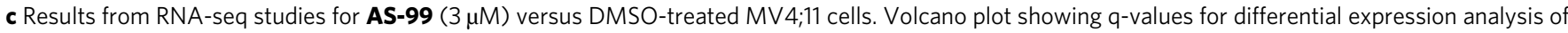
each gene versus fold-change (FC) for AS-99 over DMSO-treated cells. Blue dots indicate significantly downregulated genes, red dots indicate significantly upregulated genes $(q<0.05$, fold-change $>2$ ). d, e Plots showing enriched gene sets upon treatment of MV4;11 cells with AS-99 or DMSO determined by fgsea for defined targets of MLL-AF927 (d) or targets of NUP98-HOXA928 (e). The top 20 differentially expressed genes from the Leading Edge of the indicated fgsea analysis are shown below each of the enrichment plots. padj = adjusted $p$ value; ES enrichment score, NES normalized enrichment score. f-h Effect of AS-99 (30 mg/kg, q.d., i.p.) on leukemia burden in the MV4;11 xenotransplantation murine model. Quantification of bioluminescence signal in mice treated with AS-99 ( $n=7$ mice) or vehicle $(n=6$ mice) at the indicated days. Mean \pm SEM (f). Quantification of bioluminescence signal shown for individual mice at the last day of treatment (day 19 post-transplantation). Mean \pm SD (g). Flow cytometry quantification of human CD45+ cells in spleen, and blood samples harvested from the mice after last day of treatment with AS-99 $(n=7$ mice) or vehicle $(n=6$ mice) (h). Mean \pm SD. $P$ values were calculated using the unpaired 2-tailed $t$ test. Gating strategy is presented in Supplementary Fig. 11e.

domain (amino acids 2046-2330) was expressed and purified as reported ${ }^{21}$ and was used in the histone methyltransferase assays.

NMR-based fragment screening and compound binding to ASH1L. Samples for NMR studies were prepared with $100 \mu \mathrm{M}{ }^{15} \mathrm{~N}$-labeled ASH1L SET domain (2069-2288) in the buffer (50 mM Tris, $\mathrm{pH}=7.5,100 \mathrm{mM} \mathrm{NaCl}, 1 \mathrm{mM}$ TCEP) mixed with compounds at various concentrations $(100-500 \mu \mathrm{M})$ with a final DMSO concentration of $5 \%$. For fragment screening, we used an in-house library of 1600 fragment-like compounds with the following properties: average $\mathrm{M}_{\mathrm{w}}=183$ $\mathrm{Da}$ (range of $84-345 \mathrm{Da}$ ), mean $\operatorname{cog} \mathrm{P}=1.5$ (from -2.5 to 3.7 ), hydrogen bond acceptors: from 0 to $6($ median $=3$ ), hydrogen bond donors: 0 to 3 (median $=1)$,

Compounds were screened in mixtures of 20 compounds per sample at $250 \mu \mathrm{M}$ final concentration of each compound and 5\% DMSO. ${ }^{1} \mathrm{H}^{-15} \mathrm{~N}$ TROSY-HSQC spectra were acquired at $30^{\circ} \mathrm{C}$ on a $600 \mathrm{MHz}$ Bruker Avance III spectrometer equipped with cryoprobe, running Topspin version 2.1. Processing and spectral visualization was performed using NMRPipe v3.032 and Sparky v3.113 (T. D. Goddard and D. G. Kneller, SPARKY 3, University of California, San Francisco).

Chemical synthesis of ASH1L inhibitors. Description of chemical synthesis of ASH1L inhibitors is provided in the Supplementary Information.

Histone methyltransferase assay. Histone methyltransferase (HMT) assays were carried out in duplicates with $50 \mathrm{nM}$ ASH1L (amino acids 2046-2330) titrated by varying concentrations of compounds (final DMSO concentration was $2 \%$ ) and a buffer containing $50 \mathrm{mM}$ Tris $\mathrm{pH}=8.5,2 \mathrm{mM} \mathrm{MgCl}, 1 \mathrm{mM}$ TCEP and $0.01 \%$ Triton X-100. The mixtures of ASH1L and compounds were incubated at room temperature for $1 \mathrm{~h}$. The HMT reactions were initiated by addition of $250 \mathrm{nM}$ chicken nucleosomes (Reaction Biology, HMT-35-179), $1 \mu \mathrm{M}{ }^{3} \mathrm{H}$-labelled S-adenosyl methionine (Perkin Elmer, NET155V250UC) and $2 \mu \mathrm{M}$ unlabeled S-adenosyl methionine. The reaction was continued for $1 \mathrm{~h}$ at room temperature, then quenched by addition of $10 \%$ Trichloroacetic acid (TCA) and then transferred to 96-well filter plates (Millipore sigma, MSFBN6B), washed twice with 10\% TCA and then once with 200-proof ethanol. After extensive drying, $70 \mu \mathrm{L}$ of Microscint-O scintillant (PerkinElmer) was added and counts per minute (CPM) were measured using a MicroBeta microplate counter (PerkinElmer). Titration curves were plotted using Prism (GraphPad Prism 8.0.0.) and $\mathrm{IC}_{50}$ values were calculated.

To assess the mechanism of action of ASH1L inhibitors, the $\mathrm{IC}_{50}$ values for AS6 were determined using varying concentrations of chicken nucleosomes, $0.1 \mu \mathrm{M}$ ASH1L (2046-2330) and $40 \mu \mathrm{M}$ of SAM $\left(4 \mu \mathrm{M}{ }^{3} \mathrm{H}\right.$ SAM plus $36 \mu \mathrm{M}$ SAM). The experiments were performed in duplicates.

The effect of AS-99 on the inhibition of a panel of histone methyltransferases was assessed by mixing $50 \mu \mathrm{M}$ of the compound with ASH1L, DOT1L, EZH2, G9a, GLP, NSD1, NSD2, NSD3, MLL1, MLL4, PRDM9, PRMT1, PRMT3, PRMT4, PRMT6, SETD2, SET7/9, SYMD1, SYMD2, or SUV39. These assays were pursued under the conditions provided in Supplementary Table 3.

Isothermal titration calorimetry. Purified ASH1L (2069-2288) was extensively dialyzed against ITC buffer consisting of $50 \mathrm{mM}$ phosphate $(\mathrm{pH}=7.5), 50 \mathrm{mM}$ $\mathrm{NaCl}, 1 \mathrm{mM}$ TCEP at $4{ }^{\circ} \mathrm{C}$. Compounds were dissolved in DMSO and diluted with the ITC buffer to final concentrations of $150-500 \mu \mathrm{M}$ in 5\% DMSO. ASH1L was diluted with the ITC buffer to the final concentrations of $15-50 \mu \mathrm{M}$ in 5\% DMSO. SAM was introduced to the solutions of ASH1L and compounds to the final concentration of $50 \mu \mathrm{M}$ to maintain ASH1L stability. All samples were extensively degassed by vacuum aspiration for $20 \mathrm{~min}$ prior to measurements. The titrations were performed using a VP-ITC titration calorimetric system (MicroCal) at $25^{\circ} \mathrm{C}$. The titration curves were obtained by injecting $10 \mu \mathrm{L}$ aliquots of compound solutions into the cell containing ASH1L at a time intervals of $200 \mathrm{~s}$. For AS-85, the titration curves were obtained by injecting ASH1L $(100-130 \mu \mathrm{M})$ to the compound solutions $(10-15 \mu \mathrm{M})$. All titration data were analyzed with a single-site fitting model using Origin 7.0.

Crystallization of ASH1L-inhibitor complexes. For crystallization of ASH1L Q2265A in complex with AS-5, 7 mg/mL of ASH1L Q2265A (2069-2288) in 50 $\mathrm{mM}$ Tris $\mathrm{pH}=7.5,100 \mathrm{mM} \mathrm{NaCl}, 1 \mathrm{mM}$ TCEP buffer was mixed with AS-5 (1:1 ratio) to reach final DMSO concentration of $5 \%$. Crystals were obtained using the sitting drop method by adding the crystallization buffer containing $0.1 \mathrm{M}$ Tris $(\mathrm{pH}=8.5), 0.2 \mathrm{M} \mathrm{MgCl}_{2}$, and 30\% PEG4000 to the mixture of ASH1L Q2265Ainhibitor complex (1:1 ratio). Crystallization plates were incubated at $17^{\circ} \mathrm{C}$. For cryoprotection, crystals were soaked in the crystallization buffer solution containing 25\% PEG400 and were flash-frozen in the liquid nitrogen, prior to data collection. For crystallization of ASH1L in complex with AS-85, ASH1L (2069-2288) was concentrated to $8.4 \mathrm{mg} / \mathrm{mL}$ and was incubated with AS-85 at 1:1 molar ratio in a final DMSO concentration of $2 \%$. Crystals were obtained using the sitting drop method in $0.1 \mathrm{M}$ HEPES (pH 7.5), 30\% PEG3350 at $17^{\circ} \mathrm{C}$. For cryoprotection, crystals were soaked in a crystallization buffer containing $25 \%$ glycerol and flash-frozen in liquid nitrogen prior to data collection.

Crystallographic data collection and structure determination. X-ray diffraction data were collected under cryogenic conditions at the 21ID-F, 21ID-G beamlines of the Life Sciences Collaborative Access Team at the Advanced Photon Source. The data were then indexed, integrated, and scaled by HKL2000 v. $720^{33}$. Structures of the complexes were determined by the molecular replacement using MOLREP v.11 $1^{34}$ and Phaser-MR v.1.17.1 ${ }^{35}$ with ASH1L SET domain (PDB: 4YNM) as the search model. Refinement of the structures was performed using Phenix v.1.17.136 and Coot v.0.8.9.2 $2^{37}$. The structures were validated using MolProbity v.4.02b-467 ${ }^{38}$.

Profiling of AS-99 with kinases. Profiling of AS-99 was performed at $25 \mu \mathrm{M}$ compound concentration upon $1 \mathrm{~h}$ incubation with a panel of diverse, representative kinases at room temperature. This study was outsourced to the Contract Research Organization.

Generation of murine bone marrow cells transformed with oncogenes. Generation of C57BL6 mice with the genotype of Ash1l SET domain deletion $(\triangle \mathrm{SET})$ was previously described ${ }^{5}$. Preparation of mouse cells transformed with $M L L-A F 9$, MLL-AF6, E2A-HLF, and HOXA9/MEIS1 (HM-2) oncogenes was performed in a similar way as described previously ${ }^{39}$. Briefly, mice were injected with 5fluorouracil (Sigma, cat. \#F6627) at $150 \mathrm{mg} / \mathrm{kg}$, i.p. Five days later, the Lin-c-kit+ bone marrow cells were isolated from these mice using EasySep kit according to the manufacturer's protocol (STEMCELL, cat. \# 19856). The cells were then transduced via two rounds of spinoculation with retrovirus harvested from PlatE cells transfected with the plasmids encoding: MLL-AF9, MLL-AF6, E2A-HLF, HOXA9/ MEIS1 (HM-2), or MSCV-based vector (plasmids were kindly provided by Dr. Muntean). The transformed cells were then used for four rounds of colony forming assays with serial replating. 
Colony assay with WT and $\Delta$ SET Ash1l murine bone marrow cells. Mouse bone marrow cells transformed with various oncogenes or the vector alone were plated in the $35 \mathrm{~mm}$ dishes (StemCell, cat \# 27100) at the concentration of $1 \times 10^{4}$ cells/ $\mathrm{mL}$ in the methylcellulose medium (StemCell, cat \# M3234) containing $20 \%$ IMDM medium, $1 \%$ penicillin/streptomycin, murine IL-3 $10 \mathrm{ng} / \mathrm{ml}$, IL-6 $10 \mathrm{mg} / \mathrm{ml}$, and SCF $50 \mathrm{ng} / \mathrm{ml}$. Plates were incubated at $37^{\circ} \mathrm{C}, 5 \% \mathrm{CO}_{2}$ for $7-10$ days, at which time colonies were counted. To re-plate cells for the $2 \mathrm{nd}, 3 \mathrm{rd}$, and 4 th rounds, the cells were collected from the plates, washed in PBS twice, counted, and plated in the methylcellulose medium in the same way as in the 1st round. Colonies were counted for each round, and colonies from the 4th round were used for WrightGiemsa staining.

Viability assays. Human leukemia cells K562, MV4;11 and RS4;11 (purchased from ATCC) and KOPN8, MOLM13 (purchased from DSMZ) were cultured in RPMI 1640 (Invitrogen) supplemented with 10\% heat inactivated (h.i.) FBS and 1\% penicillin/streptomycin (Invitrogen). SET2 cells (purchased from DSMZ) were cultured in RPMI 1640 supplemented with $20 \%$ h.i. FBS and $1 \%$ penicillin/streptomycin. Cells were plated at $1 \times 10^{5}$ to $2 \times 10^{5}$ cells $/ \mathrm{mL}$ depending on cell line and treated with $0.25 \%$ DMSO or varying concentrations of compounds and incubated at $37^{\circ} \mathrm{C}, 5 \% \mathrm{CO}_{2}$ incubator for 7 days. Media was changed on day 3 or 4 and compounds were resupplied. Viable cell numbers for DMSO treated samples were restored to the original concentration and the same cell dilution was used for all other samples. Cell suspensions $(100 \mu \mathrm{L})$ were transferred to the 96 -well plates for each sample in quadruplicate at the time of media change. At day 7, $10 \mu \mathrm{L}$ of MTT reagent (M6494, Molecular Probes ${ }^{\mathrm{vt}}$ ) was added to each well of the 96 well plate and incubated at $37^{\circ} \mathrm{C}$ for $4 \mathrm{~h}$. The formazan was then solubilized by adding $100 \mu \mathrm{L}$ acidified $10 \%$ SDS solution and incubated at $37^{\circ} \mathrm{C}$ overnight. Plates were read for absorbance at $570 \mathrm{~nm}$ using a PHERAstar BMG microplate reader. The experiments were performed two times in quadruplicates with calculation of mean and SD for each condition. Data were analyzed in Prism 8.0.0 to obtain $\mathrm{GI}_{50}$ values.

Real time qRT-PCR. $0.1 \times 10^{6}$ cells/mL of MV4;11 or MOLM13 cells were plated in triplicates and treated with DMSO, AS-99 or AS-nc, maintaining the final DMSO concentration at $0.25 \%$ and were incubated at $37^{\circ} \mathrm{C}$ in a $5 \% \mathrm{CO}_{2}$ incubator. Cells were counted at day 3 or 4 using Trypan Blue (Thermo Fisher) and media was changed with compounds resupplied at that time point. Viable cell numbers for DMSO treated samples were restored to the original concentration and the same cell dilution was used for other samples. $0.5 \times 10^{6}-3 \times 10^{6}$ cells from each sample were collected at days 7 or 8 of treatment, centrifuged at $211 \mathrm{xg}$, and RNA was extracted according to the RNeasy Mini Kit protocol (74106, Qiagen). Extracted RNA was quantified using Nanodrop $2000 \mathrm{UV}$-Vis spectrophotometer and $0.5-1 \mu \mathrm{g}$ of total RNA was reverse transcribed into cDNA using High-Capacity cDNA Reverse Transcription Kit (4368814, Applied Biosystems $\mathrm{s}^{\mathrm{m}}$ ) according to the manufacturer's protocol. Real-time PCR was performed using the CFX96 Touch Real-Time PCR Detection System (Bio-Rad). TaqMan Gene Expression Master Mix and TaqMan Gene Expression Assays were purchased from Applied Biosystems. Relative quantification of each gene transcript was carried out using the comparative Ct method as described in the Applied Biosystems User Bulletin no. 2. TaqMan Gene Expression Assays were used: HPRT1: Hs02800695 m1, DLX2: Hs00269993_m1, FLT3: Hs00174690_m1, HOXA9: Hs00365956_m1, MEF2C: Hs00231149_m1, MEIS1: Hs00180020_m1, MNDA: Hs00935905_m1.

For mouse cells from WT or $\triangle$ SET Ashll background transformed with various oncogenes, cells were collected after the 4 th round of colony-forming assay, washed twice with PBS, and counted. $0.5-2 \times 10^{6}$ cells were used for RNA extraction. The RNA extraction and Real time PCR procedure were performed in a similar way as described above for human leukemia cell lines. TaqMan Gene Expression Assays were used: Gapdh: Hs99999905_ml, Hoxa9: Hs00365956_m1, Meis1: Hs00180020_m1, Hoxa5: Hs00430330_m1, Hoxa10: Hs00172012_m1, Hoxa7: Hs00600844_m1, Mef2C: Hs00231149_m1, CD11b: Mm00434455, Gr-1: Mm00439154, Ly-6G: Mm04934123.

Flow cytometry analysis. K562, MOLM13, and MV4;11 cells were plated at $1 \times$ $10^{5} \mathrm{cell} / \mathrm{mL}$ and treated for 7 or 8 days with AS-99, AS-nc or DMSO in triplicates in the same way as described for qRT-PCR studies. For Annexin V apoptosis assay, $1 \times 10^{5}$ cells per sample were collected at the end of the treatment, washed with PBS and stained using the FITC Annexin V Apoptosis Detection Kit I (BD Pharmingen ${ }^{\text {mII }}$ ) according to the manufacturer's instructions. In addition, $1 \times 10^{5}$ cells per sample were stained with Zombie Aqua ${ }^{\text {mi }}$ dye (1:100 dilution) for $15 \mathrm{~min}$ using the Zombie Aqua ${ }^{\mathrm{aw}}$ Fixable Viability Kit (Zombie Aqua ${ }^{\mathrm{at}}$ Fixable Viability Kit, Biolegend ${ }^{\circ}$ ) at 1:50 dilution and then for 30 min with an anti-CD11B-PE antibody (982606, ICRF44, Biolegend ${ }^{\circledR}$ ) or anti-CD11B-Pacific Blue (101224, BD BioLegend) antibody at 1:50 dilution, according to the No-wash Sequential Staining Protocol described by the manufacturer (BioLegend, Zombie Aqua Fixable Viability Kit). Cells were washed twice with $500 \mu \mathrm{L}$ PBS containing $1 \%$ FBS and resuspended in $200 \mu \mathrm{L}$ PBS with $1 \%$ FBS. Flow cytometry experiments were performed on FACSCelesta flow cytometer using BD FACSDiva version 8 and all data were analyzed with FlowJo v.10.6.0 software (Tree Star, Inc.).
RNA-seq studies. MV4;11 cells were plated at a concentration of $1 \times 10^{5}$ cells $/ \mathrm{mL}$ and treated with DMSO or $3 \mu \mathrm{M}$ of AS-99 for 7 days in a similar way as for qRTPCR experiments. Total RNA was prepared from cells collected after 7 days of treatment using RNeasy Mini Kit (74106, Qiagen). $2 \mu \mathrm{g}$ of total RNA for each sample, as quantified using Nanodrop 2000 UV-Vis spectrophotometer, was submitted to the Next-Generation Sequencing Services of the University of Michigan for RNA Sequencing on the Illumina NovaSeq (S4) Sequencer. ERCC RNA SpikeIn Mix (4456740, Invitrogen ${ }^{\text {tw }}$ ) was provided to control for variability in RNA expression.

For analysis of RNA-seq data, Fastq generation was performed using Illumina's bcl2fastq2 software (v2.17). The cutadapt [https://cutadapt.readthedocs.org/en/ stable/] and FastQC [http://www.bioinformatics.babraham.ac.uk/projects/fastqc/] tools wrapped in Trim Galore [http://www.bioinformatics.babraham.ac.uk/ projects/trim_galore/] were used to trim low quality bases $(\mathrm{Q}<20)$ and adapter for raw sequences. Reads with length $<20$ bp were removed after trimming. Cleaned RNA-seq reads were mapped to the hg19 genome for human using Tophat2 $(2.1 .1)^{40}$, which was shown to be accurate alignment of transcriptomes in the presence of insertions, deletions, and gene fusions. Duplicated reads for pair-end data were removed by SAMtools $(\mathrm{v} 1.5)^{41}$. Aligned reads were assembled using Cufflinks (v2.2.1) and assembled transcriptome catalog was used as an input for Cuffdiff ${ }^{42}$ to determine gene expression levels (FPKM, Fragments Per Kilobase per Million mapped reads) and differential expression between conditions using default options. Genes with $p<0.05$ and fold-change $>2$ up or down were considered as differentially expressing genes to prepare the Volcano plot, which was generated using the "ggplot2" R package. Gene set analysis was performed and enrichment plots were generated using the "fgsea" package, with the test statistic generated by Cuffdiff 2 used to rank genes for gene set enrichment analysis ${ }^{43}$. Fgsea was run using the $\mathrm{C} 2, \mathrm{C} 5$, and $\mathrm{C} 6$ curated datasets from $\mathrm{MSigDB}^{44}$. Heatmaps for the top 20 ranked genes in the Leading Edge from a given enrichment analysis were generated with the "gplots" package [https://cran.r-project.org/web/packages/ gplots/index.html].

Cytospins and Wrigth-Giemsa staining. 0.1 to $1 \times 10^{5}$ cells treated with AS-99, AS-nc or $0.25 \%$ DMSO were collected at day 7 for staining. Cytospins were prepared and stained using the PROTOCOL ${ }^{\text {nx }}$ Hema $3^{\text {max }}$ Manual Staining System (22122911, Fisher Scientific) as described before ${ }^{45}$. For WT or $\Delta$ SET Ash1l murine bone marrow cells transformed with various oncogenes, cells were collected after fourth round of colony assay, washed twice with PBS, and counted. 0.5 to $1 \times 10^{5}$ cells in $100 \mu \mathrm{L}$ PBS were used for cytospins and staining by applying the same procedure as described before ${ }^{45}$.

CUT\&RUN experiment. MV4;11 cells were plated at $1 \times 10^{5} \mathrm{cells} / \mathrm{mL}$ and treated with $0.25 \%$ DMSO or $5.5 \mu \mathrm{M}$ AS-99 in triplicates for 4 days. At day 4 , cells were counted using Trypan Blue dye, centrifuged at $211 \mathrm{xg}$ and washed with $\mathrm{Ca}^{++}$and $\mathrm{Mg}^{++}$free 1X DPBS (14190144, Gibco $\left.{ }^{\mathrm{m}}\right) .2 \times 10^{6}$ viable cells per sample per antibody were processed for CUT\&RUN experiment as previously described ${ }^{46}$ Briefly, cells were collected and washed by PBS at room temperature. $2 \times 10^{6}$ cells were incubated with $30 \mu \mathrm{L}$ Concanavalin A-coated beads (Bangs Laboratories, cat. \# BP531) at room temperature for $10 \mathrm{~min}$ followed by overnight incubation at $4{ }^{\circ} \mathrm{C}$ with antibody against H3K36me2 (39255, Active Motif, $4 \mu \mathrm{g} / \mathrm{mL}$ ) at $4{ }^{\circ} \mathrm{C}$. Samples were washed with DIG-wash buffer and treated with Protein A-micrococcal nuclease (pA-MN) fusion protein to a final concentration of $1 \mu \mathrm{g} / \mathrm{mL}$ at room temperature for $10 \mathrm{~min}$. Digestion was carried out in the presence of $2 \mathrm{mM} \mathrm{CaCl}_{2}$ for $30 \mathrm{~min}$ at $0{ }^{\circ} \mathrm{C}$. The digested and released particles were extracted by organic extraction method using phenol, chloroform, and ethanol. Purified DNA was submitted to the Next Generation Sequencing Services at the University of Michigan for sequencing on the Illumina NovaSeq (S4) Sequencer. Spike-in DNA (Micrococcal nuclease-treated Drosophila chromatin) was used for calibration.

For analysis of CUT\&RUN data, cleaned sequencing reads were mapped to the hg19 genome for human by using Bowtie2 (v2-2.2.4) ${ }^{47}$ with parameters "-q --phred 33 --end-to-end --very-sensitive --no-mixed --no-discordant --phred33 -I 10 -X 700". Then, duplicated reads were removed using SAMtools (v1.5) ${ }^{41}$. Fragments of sizes $>120$ bp for H3K36me2 CUT\&RUN were selected for downstream analysis. Filtered BAM files of mapping results were merged for the same sample using SAMtools and converted to BED format by using BEDTools $\mathrm{v} 2.28 .0^{48}$. Peak regions were called for each sample by using MACS (v 1.4.2) ${ }^{49}$ from bed files of Cut\&Run with parameters "-w -S -p 0.00001 -g hs". RPKM values for visualization in IGV v2.350 were generated by MACS (V 1.4.2) ${ }^{49}$. Differential peaks between groups were called by MACS2 V2.1.1 ${ }^{51}$ subcommand "bdgdiff". Homer $\mathrm{v} 4.10^{52}$ was employed to perform gene annotation of peaks and differential peaks. Heatmap of signals of H3K36me2 was plotted by deepTools v2.5.753. Violin plots of $\mathrm{H} 3 \mathrm{~K} 36 \mathrm{me} 2$ were performed by R package ggpubr v0.4.0.

PK studies in mice. The pharmacokinetics of AS-99 ASH1L inhibitor was determined in female CD-1 mice (Charles River) following intravenous (i.v.) at 15 $\mathrm{mg} / \mathrm{kg}$ and Intraperitoneal (i.p.) at $30 \mathrm{mg} / \mathrm{kg}$ dosing. AS-99 was dissolved in the vehicle containing 25\% DMSO, 45\% PEG 400 and PBS. Serial blood samples $(70 \mu \mathrm{L})$ were collected over $24 \mathrm{~h}$, centrifuged at $21130 \mathrm{xg}$ for $10 \mathrm{~min}$ to get plasma for analysis. Plasma concentrations of AS-99 were determined by the LC-MS/MS 
method developed and validated for this study. The LC-MS/MS method consisted of a Shimadzu UFLC 20 A system and chromatographic separation of test compound was achieved using Waters XBridge C18 column $(5 \mathrm{~cm} \times 2.1 \mathrm{~mm}, 3.5 \mu \mathrm{m})$. An AB Sciex QTrap 5500 mass spectrometer equipped with an electrospray ionization source (ABI-Sciex, Toronto, Canada) in the positive-ion multiple reaction monitoring (MRM) mode was used for detection. All pharmacokinetic parameters were calculated by noncompartmental methods using WinNonlin ${ }^{\oplus}$ version 3.2 (Pharsight Corporation, Mountain View, CA, USA). Parameters are presented as a mean \pm standard deviation $(\mathrm{SD})$.

In vivo studies using bioimaging model. The in vivo bioimaging model was developed as described previously ${ }^{29,54}$. Briefly, the 8- to 10 -week old female NSG mice (Jackson Laboratory) were intravenously injected with $5 \times 10^{6}$ luciferaseexpressing MV4;11 cells (received from Dr. Thomas Look, Dana Farber Cancer Institute). On day 6 after transplantation, mice were split into vehicle $(n=6)$ and AS-99 $(n=7)$ treatment groups and treated for 14 consecutive days with vehicle (25\% DMSO and 25\% PEG400 in PBS) or AS-99 (30 mg $/ \mathrm{kg}$, q.d., i.p.). For imaging, mice were kept anesthetized with isoflurane, and $50 \mathrm{mg} / \mathrm{kg}$ luciferin (E1603, Promega) was administered by intraperitoneal injection. Photonic emission was imaged using the in Vivo Imaging System BLI (IVIS, 200) with total imaging time of $10 \mathrm{~s}$ for each mouse.

Flow cytometry analysis of hCD45 + cells. Cells isolated from spleen or peripheral blood (PB) of mice treated with AS-99 (30 mg/kg, q.d., i.p.) or vehicle were used for the analysis of the level of human $\mathrm{CD} 45+(\mathrm{hCD} 45+)$ cells. Red blood cells were lysed with ACK lysis buffer (10-548E, Lonza). Up to 100,000 cells were washed in PBS (with 1\% FBS) and stained with the anti-human CD45-BV421 antibody (563879, HI30, BD Biosciences) at 1:50 dilution for $30 \mathrm{~min}$. Flow cytometry experiments were performed on LSR II, FACSCanto, or FACSAria (BD Biosciences) instruments and data were analyzed with FlowJo v.10.6.0 software (Tree Star, Inc.)

Effect of AS-99 on normal hematopoiesis. For analysis of the effect of AS-99 on normal hematopoiesis in vivo, 8-10 weeks old female C57BL/6 mice (The Jackson Laboratory) were treated with vehicle $(n=9)$ or AS-99 $(n=9)(30 \mathrm{mg} / \mathrm{kg}$, i.p., q.d. $)$ for 14 days. At the end point of the experiment blood samples were collected, processed and analyzed for complete blood counts $(\mathrm{CBC})$ and to assess populations of myeloid $(\mathrm{CD} 11 \mathrm{~b}+/ \mathrm{Gr}-1+)$, B- $(\mathrm{B} 220+/ \mathrm{CD} 19+)$, and $\mathrm{T}-(\mathrm{CD} 3+)$ cells using flow cytometry. The following antibodies from BioLegend were used for staining: anti-CD11b (\#101208, M1/70), Gr-1 (\#108433, RB6-8C5), B220 (\#103224, RA3-6B2), CD19 (\#115520, 6D5), CD3 (\#109211, H57-597), Zombie Aqua-dead cells (\#423101) at 1:50 dilution. The analysis of CBC and other blood parameters was performed by the ULAM Animal Diagnostic Laboratory using Element HT5 (HESKA).

Ethics. Animal experiments performed in this study were approved by the University of Michigan Committee on Use and Care of Animals (UCUCA) and Unit for Laboratory Animal Medicine (ULAM). All mouse studies included randomization and were not blinded.

Reporting summary. Further information on research design is available in the Nature Research Reporting Summary linked to this article.

\section{Data availability}

The data that support this study are available from the corresponding authors upon reasonable request. The coordinates for ASH1L-AS-5 and ASH1L-AS-85 complexes were deposited in PDB under PDB codes 6X0P [https://doi.org/10.2210/pdb6x0p/pdb] and 6WZW [https://doi.org/10.2210/pdb6wzw/pdb], respectively. RNA-seq and CUT\&RUN data were deposited to GEO under the accession number GSE150087 (SubSeries numbers: GSE150085 and GSE150086 for the RNA-seq and CUT\&RUN data, respectively). Source data are provided with this paper.

Received: 14 August 2020; Accepted: 13 April 2021; Published online: 14 May 2021

\section{References}

1. Dawson, M. A. \& Kouzarides, T. Cancer epigenetics: from mechanism to therapy. Cell 150, 12-27 (2012).

2. Rogawski, D. S., Grembecka, J. \& Cierpicki, T. H3K36 methyltransferases as cancer drug targets: rationale and perspectives for inhibitor development. Future Med Chem. 8, 1589-1607 (2016).

3. Wagner, E. J. \& Carpenter, P. B. Understanding the language of Lys 36 methylation at histone H3. Nat. Rev. Mol. Cell Biol. 13, 115-126 (2012).
4. Jones, M. et al. Ash1l controls quiescence and self-renewal potential in hematopoietic stem cells. J. Clin. Invest. 125, 2007-2020 (2015).

5. Miyazaki, H. et al. Ash1l methylates Lys 36 of histone $\mathrm{H} 3$ independently of transcriptional elongation to counteract polycomb silencing. PLoS Genet $\mathbf{9}$, e1003897 (2013).

6. Gregory, G. D. et al. Mammalian ASH1L is a histone methyltransferase that occupies the transcribed region of active genes. Mol. Cell Biol. 27, 8466-8479 (2007).

7. Rogawski, D. S. et al. Two loops undergoing concerted dynamics regulate the activity of the ASH1L histone methyltransferase. Biochemistry 54, 5401-5413 (2015).

8. Schuettengruber, B., Chourrout, D., Vervoort, M., Leblanc, B. \& Cavalli, G. Genome regulation by polycomb and trithorax proteins. Cell 128, 735-745 (2007).

9. Zhu, L. et al. ASH1L links histone H3 lysine 36 dimethylation to MLL leukemia. Cancer Disco. 6, 770-783 (2016).

10. Tanaka, Y., Katagiri, Z., Kawahashi, K., Kioussis, D. \& Kitajima, S. Trithoraxgroup protein ASH1 methylates histone H3 lysine 36. Gene 397, 161-168 (2007).

11. McMahon, K. A. et al. Mll has a critical role in fetal and adult hematopoietic stem cell self-renewal. Cell Stem Cell 1, 338-345 (2007).

12. Trissal, M. C. et al. MIR142 loss-of-function mutations derepress ASH1L to increase HOXA gene expression and promote leukemogenesis. Cancer Res. 78, 3510-3521 (2018).

13. Cox, M. C. et al. Chromosomal aberration of the 11q23 locus in acute leukemia and frequency of MLL gene translocation: results in 378 adult patients. Am. J. Clin. Pathol. 122, 298-306 (2004).

14. Sorensen, P. H. et al. Molecular rearrangements of the MLL gene are present in most cases of infant acute myeloid leukemia and are strongly correlated with monocytic or myelomonocytic phenotypes. J. Clin. Invest. 93, 429-437 (1994).

15. Pui, C. H. et al. Outcome of treatment in childhood acute lymphoblastic leukaemia with rearrangements of the $11 \mathrm{q} 23$ chromosomal region. Lancet 359, 1909-1915 (2002).

16. Colamaio, M. et al. miR-142-3p down-regulation contributes to thyroid follicular tumorigenesis by targeting ASH1L and MLL1. J. Clin. Endocrinol. Metab. 100, E59-E69 (2015).

17. Liu, L., Kimball, S., Liu, H., Holowatyj, A. \& Yang, Z. Q. Genetic alterations of histone lysine methyltransferases and their significance in breast cancer. Oncotarget 6, 2466-2482 (2015)

18. Fujimoto, A. et al. Whole-genome mutational landscape and characterization of noncoding and structural mutations in liver cancer. Nat. Genet. 48, 500-509 (2016).

19. Ayton, P. M. \& Cleary, M. L. Transformation of myeloid progenitors by MLL oncoproteins is dependent on Hoxa7 and Hoxa9. Genes Dev. 17, 2298-2307 (2003).

20. Fick, R. J. et al. Sulfur-oxygen chalcogen bonding mediates AdoMet recognition in the lysine methyltransferase SET7/9. ACS Chem. Biol. 11, 748-754 (2016).

21. Eram, M. S. et al. Kinetic characterization of human histone H3 lysine 36 methyltransferases, ASH1L and SETD2. Biochim. Biophys. Acta 1850, 1842-1848 (2015)

22. Hopkins, A. L., Keseru, G. M., Leeson, P. D., Rees, D. C. \& Reynolds, C. H. The role of ligand efficiency metrics in drug discovery. Nat. Rev. Drug Disco. 13, 105-121 (2014).

23. Qiao, Q. et al. The structure of NSD1 reveals an autoregulatory mechanism underlying histone H3K36 methylation. J. Biol. Chem. 286, 8361-8368 (2011)

24. Daigle, S. R. et al. Potent inhibition of DOT1L as treatment of MLL-fusion leukemia. Blood 122, 1017-1025 (2013).

25. Knutson, S. K. et al. Selective inhibition of EZH2 by EPZ-6438 leads to potent antitumor activity in EZH2-mutant non-Hodgkin lymphoma. Mol. Cancer Ther. 13, 842-854 (2014).

26. Armstrong, S. A. et al. MLL translocations specify a distinct gene expression profile that distinguishes a unique leukemia. Nat. Genet. 30, 41-47 (2002).

27. Kumar, A. R. et al. Hoxa9 influences the phenotype but not the incidence of Mll-AF9 fusion gene leukemia. Blood 103, 1823-1828 (2004).

28. Takeda, A., Goolsby, C. \& Yaseen, N. R. NUP98-HOXA9 induces long-term proliferation and blocks differentiation of primary human $\mathrm{CD} 34+$ hematopoietic cells. Cancer Res. 66, 6628-6637 (2006).

29. Borkin, D. et al. Pharmacologic inhibition of the Menin-MLL interaction blocks progression of MLL leukemia in vivo. Cancer Cell 27, 589-602 (2015).

30. Brinkmeier, M. L. et al. The histone methyltransferase gene absent, small, or homeotic discs-1 like is required for normal hox gene expression and fertility in mice. Biol. Reprod. 93, 121 (2015).

31. DelProposto, J., Majmudar, C. Y., Smith, J. L. \& Brown, W. C. Mocr: a novel fusion tag for enhancing solubility that is compatible with structural biology applications. Protein Expr. Purif. 63, 40-49 (2009).

32. Delaglio, F. et al. NMRPipe: a multidimensional spectral processing system based on UNIX pipes. J. Biomol. NMR 6, 277-293 (1995). 
33. Otwinowski, Z. \& Minor, W. Processing of X-ray diffraction data collected in oscillation mode. Methods Enzymol. 276, 307-326 (1997).

34. Vagin, A. \& Teplyakov, A. Molecular replacement with MOLREP. Acta Crystallogr. D. Biol. Crystallogr. 66, 22-25 (2010).

35. McCoy, A. J. et al. Phaser crystallographic software. J. Appl. Crystallogr. 40 658-674 (2007).

36. Adams, P. D. et al. PHENIX: a comprehensive Python-based system for macromolecular structure solution. Acta Crystallogr. D. Biol. Crystallogr. 66 , 213-221 (2010).

37. Emsley, P. \& Cowtan, K. Coot: model-building tools for molecular graphics. Acta Crystallogr. D. Biol. Crystallogr. 60, 2126-2132 (2004).

38. Chen, V. B. et al. MolProbity: all-atom structure validation for macromolecular crystallography. Acta Crystallogr. D. Biol. Crystallogr. 66, 12-21 (2010).

39. Muntean, A. G. et al. MLL fusion protein-driven AML is selectively inhibited by targeted disruption of the MLL-PAFc interaction. Blood 122, 1914-1922 (2013).

40. Kim, D. et al. TopHat2: accurate alignment of transcriptomes in the presence of insertions, deletions and gene fusions. Genome Biol. 14, R36 (2013).

41. Li, H. et al. The sequence alignment/map format and SAMtools. Bioinformatics 25, 2078-2079 (2009).

42. Trapnell, C. et al. Differential gene and transcript expression analysis of RNA-seq experiments with TopHat and Cufflinks. Nat. Protoc. 7, 562-578 (2012).

43. Sergushichev, A. An algorithm for fast preranked gene set enrichment analysis using cumulative statistic calculation. BioRxiv (2016). https://doi.org/10.1101/ 060012

44. Liberzon, A. et al. Molecular signatures database (MSigDB) 3.0. Bioinformatics 27, 1739-1740 (2011).

45. Grembecka, J. et al. Menin-MLL inhibitors reverse oncogenic activity of MLL fusion proteins in leukemia. Nat. Chem. Biol. 8, 277-284 (2012).

46. Li, Q. et al. p53 integrates temporal WDR5 inputs during neuroectoderm and mesoderm differentiation of mouse embryonic stem cells. Cell Rep. 30, 465-480 e466 (2020).

47. Langmead, B. \& Salzberg, S. L. Fast gapped-read alignment with Bowtie 2. Nat. Methods 9, 357-359 (2012).

48. Quinlan, A. R. \& Hall, I. M. BEDTools: a flexible suite of utilities for comparing genomic features. Bioinformatics 26, 841-842 (2010).

49. Zhang, Y. et al. Model-based analysis of ChIP-Seq (MACS). Genome Biol. 9 , R137 (2008)

50. Thorvaldsdottir, H., Robinson, J. T. \& Mesirov, J. P. Integrative Genomics Viewer (IGV): high-performance genomics data visualization and exploration. Brief. Bioinform 14, 178-192 (2013).

51. Feng, J., Liu, T., Qin, B., Zhang, Y. \& Liu, X. S. Identifying ChIP-seq enrichment using MACS. Nat. Protoc. 7, 1728-1740 (2012).

52. Heinz, S. et al. Simple combinations of lineage-determining transcription factors prime cis-regulatory elements required for macrophage and B cell identities. Mol. Cell 38, 576-589 (2010).

53. Ramirez, F. et al. deepTools2: a next generation web server for deepsequencing data analysis. Nucleic Acids Res. 44, W160-W165 (2016).

54. Klossowski, S. et al. Menin inhibitor MI-3454 induces remission in MLL1rearranged and NPM1-mutated models of leukemia. J. Clin. Invest. 130, 981-997 (2020).

\section{Acknowledgements}

We thank Dr. Thomas Look, Dana Farber Cancer Institute, for providing MV4;11 cells expressing luciferase, Dr. Masoud Vedadi, University of Toronto, for providing ASH1L construct and Dr. Raymond Trievel, University of Michigan, for providing histone methyltransferases constructs. We thank Dr. Gloria Jih for breeding ASH1L $\triangle$ SET mice. We thank Dr. John Bushweller and Dr. Olga Guryanova for providing human leukemia cell lines for this project. The authors thank Miranda Simes for critical reading of this paper. The mouse work was performed under oversight of UCUCA at the University of Michigan. This work was funded by the National Institute of Health (NIH) R01 grants
(1R01 CA244254, 1R01 CA201204 and 5R01 CA160467) to J.G., NIH R01 grants (1R01 CA226759 and 1R01 CA207272) to T. C., NIH R01 grant (1R01 AG050509) to I. M., LLS Scholar (1215-14) to J.G., LLS Scholar to T.C., LLS TRP grants 6485-16 and 6579-20 to J. G., and 6111-14 and 6564-19 to T.C., and LLS Screen to Lead grant (8017-20). James Ropa is a recipient of NIH T32 training grant (T32DK007519). This research used resources of the Advanced Photon Source, a U.S. Department of Energy (DOE) Office of Science User Facility operated for the DOE Office of Science by Argonne National Laboratory under Contract No. DE-AC02-06CH11357. Use of the LS-CAT Sector 21 was supported by the Michigan Economic Development Corporation and the Michigan Technology Tri-Corridor (Grant 085P1000817).

\section{Author contributions}

D.R., J.C., and H.L. designed the experiments, performed the majority of experiments, analyzed data, made figures and edited the manuscript. J.D., D.B., J.S., S.L., S. Klossowski, M.K. and Z.J. designed and synthesized compounds. D.R., H.L., and J.N. designed and performed in vitro biochemical, biophysical, and crystallography experiments. H.M., T.P E.K., P.E., A.M., and B.Z. designed and performed cell-biology experiments. J.C., G.H., X.F, K.N., S. Kelly., and I.M. generated and provided $\triangle$ SET Ashll mice and/or performed analysis of mice samples. J.R., F.M., and Y.D. performed RNA-seq and/or CUT\&RUN data analysis. M.H., B.W., and D.S. designed and performed PK studies. P.E., I.M., A.M., J.G., and T.C. contributed to the design and discussion of experiments. D.R., J.D., H.L., I.M., T.C., and J. G., interpreted the data and contributed to writing of the paper. J.G and T.C. directed the entire project, designed the experiments, analyzed the results and wrote the paper with the input from all authors.

\section{Competing interests}

J.G., T.C., D.R., H.L., J. D., S.K., H.M., T.P., E.K., M.S., D.B., and J. Z. are co-inventors on patent applications covering ASH1L inhibitors. T.C. and J.G. received prior research support from Kura Oncology Inc. for unrelated project; served as consultants in Kura Oncology and have equity ownership in the company. The remaining authors declare that they have no competing interests with this study.

\section{Additional information}

Supplementary information The online version contains supplementary material available at https://doi.org/10.1038/s41467-021-23152-6.

Correspondence and requests for materials should be addressed to T.C. or J.G.

Peer review information Nature Communications thanks Jian Jin, Konstantinos Tzelepis and the other, anonymous, reviewer(s) for their contribution to the peer review of this work

Reprints and permission information is available at http://www.nature.com/reprints

Publisher's note Springer Nature remains neutral with regard to jurisdictional claims in published maps and institutional affiliations.

Open Access This article is licensed under a Creative Commons Attribution 4.0 International License, which permits use, sharing, adaptation, distribution and reproduction in any medium or format, as long as you give appropriate credit to the original author(s) and the source, provide a link to the Creative Commons license, and indicate if changes were made. The images or other third party material in this article are included in the article's Creative Commons license, unless indicated otherwise in a credit line to the material. If material is not included in the article's Creative Commons license and your intended use is not permitted by statutory regulation or exceeds the permitted use, you will need to obtain permission directly from the copyright holder. To view a copy of this license, visit http://creativecommons.org/ licenses/by/4.0/

(C) The Author(s) 2021 